\title{
Indian Hedgehog release from renal epithelia drives local and remote organ fibrosis
}

David Ferenbach ( $\square$ David.Ferenbach@ed.ac.uk)

The University of Edinburgh https://orcid.org/0000-0002-9202-1428

\section{Eoin O'Sullivan}

The University of Edinburgh

\section{Katie Mylonas}

The University of Edinburgh

\section{Cuiyan Xin}

Harvard Medical School

\section{Cyril Carvalho}

The University of Edinburgh

Julia Wilflingseder

University of Veterinary Medicine https://orcid.org/0000-0002-0230-9349

Michaela Willi

$\mathrm{NIH}$

\section{Sarah Finnie}

The University of Edinburgh

\section{Angela Oliveira Pisco}

Chan Zuckerberg Biohub https://orcid.org/0000-0003-0142-2355

\section{Neil Henderson}

University of Edinburgh https://orcid.org/0000-0002-2273-4094

\section{Bryan Conway}

The University of Edinburgh

\section{Laura Denby}

The University of Edinburgh

\section{Jeremy Hughes}

The University of Edinburgh

\section{Joseph Bonventre}

Brigham and Women's Hospital https://orcid.org/0000-0001-7144-386X

\section{Biological Sciences - Article}

Keywords: chronic kidney disease, aging, organ fibrosis, cardiovascular disease, renal leukocytes, therapy 
Posted Date: September 8th, 2021

DOl: https://doi.org/10.21203/rs.3.rs-793155/v1

License: (c) (i) This work is licensed under a Creative Commons Attribution 4.0 International License. Read Full License 
Title: Indian Hedgehog release from renal epithelia drives local and remote organ

\section{fibrosis}

\section{Authors}

Eoin D O’Sullivan ${ }^{1 \circ}$, Katie J Mylonas ${ }^{1 \circ}$, Cuiyan Xin ${ }^{2 \circ}$, Cyril Carvalho ${ }^{1}$, Julia

Wilflingseder ${ }^{2,3}$, Michaela Willi ${ }^{4}$, Sarah Finnie ${ }^{5}$, Angela Pisco ${ }^{6}$, Neil C Henderson ${ }^{1,7}$, Bryan

R Conway $^{5}$, Laura Denby ${ }^{5}$, Jeremy Hughes ${ }^{1}$, Joseph V Bonventre ${ }^{2}$, David A Ferenbach ${ }^{1,2 *}$

\section{Affiliations}

${ }^{1}$ Centre for Inflammation Research, Queen's Medical Research Institute, University of Edinburgh, Edinburgh, UK.

${ }^{2}$ Renal Division and Division of Engineering in Medicine, Brigham and Women's Hospital, Department of Medicine, Harvard Medical School, Boston, MA, USA.

${ }^{3}$ Department of Physiology and Pathophysiology, University of Veterinary Medicine, Veterinärplatz 1, 1210 Vienna, Austria

${ }^{4}$ Laboratory of Genetics and Physiology, NIDDK, NIH, Bethesda, USA

${ }^{5}$ Centre for Cardiovascular Science, Queen's Medical Research Institute, University of Edinburgh, Edinburgh, UK

${ }^{6}$ Chan Zuckerberg Biohub, San Francisco, CA, USA

${ }^{7} \mathrm{MRC}$ Human Genetics Unit, Institute of Genetics and Cancer, University of Edinburgh, Edinburgh, UK

${ }^{\circ}$ denotes equal contribution

*Corresponding author 


\begin{abstract}
Chronic kidney disease (CKD) and ageing inhibit tissue regeneration and increase risks of organ fibrosis and cardiovascular disease. Increased numbers of leukocytes are present in the circulation and within the kidney of ageing individuals and patients with $\mathrm{CKD}$ where they correlate with progressive fibrosis. The involvement of activated leukocytes in progressive renal and systemic fibrosis remains incompletely understood. Here we show that renal leukocyte derived tumour necrosis factor alpha $(\mathrm{TNF} \alpha)$ promotes renal and cardiac fibrosis via downstream induction of Indian Hedgehog (IHH). We identify the Ubiquitin D expressing 'inflammatory' proximal tubular epithelia (iPT) population responsible for $\mathrm{TNF} \alpha / \mathrm{NF} \kappa \mathrm{B}$ induced IHH production. iPT cells are upregulated in the kidney in experimental murine and human renal disease and ageing. iPT derived IHH activates canonical Hedgehog signalling in Gli1+ stromal cells, inducing their activation, proliferation and fibrosis in the surrounding kidney and in remote organs including the heart. This can be inhibited by selective genetic Ihh deletion from Pax8 expressing renal epithelia, or pharmacological blockade of TNF $\alpha, \mathrm{NF} \kappa \mathrm{B}$ or Hedgehog signalling. This data connects inflammation to progressive cardiac and renal fibrosis and identifies new targets for anti-fibrotic therapies.
\end{abstract}




\section{Introduction}

'Physiological' ageing leads to reduced adaptive immunity ${ }^{1,2}$ and increased expression of mediators of innate immune activation including IL- 6 and TNF $\alpha^{3,4}$. Immune activation is also present in multiple chronic illnesses which become more common with ageing, including chronic kidney disease $(\mathrm{CKD})^{5-7}$. Of note, patients with $\mathrm{CKD}$ exhibit accelerated cardiovascular disease and fibrosis, with CKD implicated in 'premature cardiovascular ageing, ${ }^{8}$. Furthermore, the persistence of activated leukocytes within the injured kidney correlates with progressive fibrosis in animal disease models ${ }^{9}$ and human $\mathrm{CKD}^{10}$, even when the initiating insult was not immunologically mediated or has apparently resolved, such as acute kidney injury ${ }^{6}$. Thus, it has been proposed that activated leukocytes contribute to maladaptive repair, kidney fibrosis and cardiovascular disease in both ageing and $\mathrm{CKD}^{11}$. The pathways linking leukocyte activation to kidney fibrosis and cardiovascular disease remain incompletely understood. Identifying the key mechanisms which regulate this kidneycardiovascular disease axis should allow the rational design of potent therapies to treat this major healthcare burden.

Evidence implicates renal epithelia as regulators of injury outcome via interactions with leukocytes, endothelia and myofibroblasts ${ }^{12-14}$. Here we test the hypothesis that changes in the renal epithelial phenotype links ageing and inflammation to systemic fibrosis. 


\section{Results}

\section{$\mathrm{Ubd}^{+}$renal epithelia are present in aged and fibrotic kidneys in mice and humans}

To investigate whether distinct epithelial populations capable of fibrotic signalling exist in kidneys after injury we performed single cell RNA sequencing (scRNAseq) of control and fibrotic kidneys from young (6-8 week old) mice 6 weeks after ischaemia-reperfusion injury (IRI), where maladaptive repair has led to fibrosis (Fig. 1a). Given the association between ageing and renal fibrosis we examined the Tabula Muris Senis (TMS) database ${ }^{15}$ for ageingassociated renal epithelial clusters (Fig. 1a).

Control and fibrotic kidneys from young animals were analysed (Extended Data Fig 1a-d), with one proximal tubular cluster unique to the fibrotic kidney classified as "inflammatory-fibrotic" proximal tubules (iPT) based on its Differentially Expressed Gene (DEG) expression (Fig. 1bd, Extended Data 2a-b).

The presence of iPTs in uninjured ageing kidneys was confirmed using 21,647 renal cells from the TMS dataset (Fig. 1b). On epithelial-restricted analysis an ageing-enriched cluster was noted (1.4\% of epithelia in 1-3 month vs $12 \%$ in $18-30$ month-old mice) which shared DEGs with post-injury iPTs (Fig. 1b-d, Extended Data Fig. 2c). Multiple transcriptional differences separated iPT clusters from other epithelia in fibrotic and aged kidneys (Fig. 1b-d, Extended Data Fig. 2a-c). The 3 most marked DEG changes in iPT cells were increased expression of Ubiquitin D ( $U b d$, necessary for TNF $\alpha$ mediated activation in vivo $\left.{ }^{16,17}\right)$, Prostaglandin D2 Synthase (Ptgds) and CCN Family Member 1 (CCN1/Cyr61, associated with fibrosis, apoptosis and senescence ${ }^{18}$ ). iPT cells also expressed increased $V$ cam1, a marker proposed for renal epithelia with increased activation of the NFkB transcription factor ${ }^{19}$. 
Taking UBD as a key marker of iPT cells we explored human datasets for evidence of altered $U B D$ expression in the kidney with ageing. Work by Rodwell et $\mathrm{al}^{20}$ showed a positive association between increasing age and renal UBD expression. Levels of UBD were increased in human kidneys with the highest levels of interstitial fibrosis, glomerular and tubular atrophy (Extended Data Fig 3).

\section{TNF $\alpha$ induces iPT cell signalling via NFKB activation in vivo}

The presence of iPT cells in uninjured aged kidneys demonstrated that this cellular phenotype did not simply represent a response to acute kidney injury. Upregulated Ubd and Cxcll6 expression were both consistent with iPT cells responding to TNF $\alpha$ stimulation ${ }^{21}$. Further analysis of both datasets using published TNFo-induced genes from other tissues ${ }^{22}$ showed upregulation of multiple TNF $\alpha$ targets in iPT cells in vivo (Fig. 1c). In keeping with published work identifying leukocytes as the major source of renal $\mathrm{TNF}^{23}, \operatorname{Tn} f$ transcript expression in control and fibrotic kidneys was confined to leukocytes in young fibrotic kidneys (Extended Data Fig. 4). Over representation analysis in the kidney demonstrated enrichment of TNF $\alpha$ activation and its downstream NFאB signalling pathway in iPT clusters (Fig. 1d). We therefore explored the potential pathogenic role of $\mathrm{TNF} \alpha$ and $U b d+$ iPT cells in ageing and injuryinduced kidney fibrosis.

\section{$\mathrm{TNF} \alpha / \mathrm{NFkB}$ signalling induces $\mathrm{Ubd}$ expression in renal epithelia in vitro and is profibrotic in vivo}

To determine whether NFkB signalling was essential for TNF $\alpha$ mediated $U b d$ activation, human renal proximal tubular cells (RPTECs) were cultured in vitro $\pm \mathrm{TNF} \alpha \pm \mathrm{NF \kappa B}$ inhibitor 
BAY 11-7082 (Extended Data Fig. 5a). TNF $\alpha$ exposure and intact NFkB signalling were necessary for RPTEC Ubd upregulation (Extended Data Fig. 5b).

To dissect the role of TNF $\alpha$ in fibrosis we tested TNF $\alpha$ antagonism using the clinically licenced agent Infliximab in two models of renal injury and fibrosis (Fig. 2a). Consistent with a profibrotic role for $\mathrm{TNF} \alpha$, mice had less interstitial fibrosis and increased renal mass after IRI with TNF $\alpha$ antagonism (Fig. 2b). In unilateral ureteric obstruction (UUO) in aged mice TNF $\alpha$ antagonism also educed tissue fibrosis (Fig. 2c). In situ hybridisation (ISH) to Ubd and Collal demonstrated upregulation of $U b d$ in proximal tubules after injury. $U b d+$ cell number was reduced five-fold to baseline by TNF $\alpha$ antagonism, consistent with elevated levels of TNFa inducing $U b d+$ iPT cells in vivo (Fig. $2 \mathrm{~d}$ ). Collectively, these findings support the iPT cluster representing epithelia undergoing $\mathrm{TNF} \alpha$ mediated activation of $\mathrm{NF \kappa B}$ transcription in vivo.

TNF $\alpha$ induced NFKB activation results in Ihh transcription by iPT cells in vitro and in vivo

To determine the mechanism connecting TNF $\alpha$ to tissue fibrosis we returned to our scRNAseq datasets to assess pathways known to be activated in renal disease ${ }^{24}$. Analysis was undertaken on Wnt, Hedgehog (Hh) and TGF- ligand expression in iPT cells from fibrotic and aged kidneys compared to all other epithelial clusters (Fig. 1b and online data). No upregulation was seen in any $W n t$ ligand. $T g f b 1, T g f b 2$ and Indian Hedgehog $(I h h)$ transcripts were all differentially expressed and upregulated in iPT cells. Although published work implicated epithelial Sonic Hedgehog $(S h h)$ induction as a key driver of kidney fibrosis in vivo ${ }^{25}$, $I h h$ was the sole Hedgehog transcript expressed at high levels by any renal cell (Fig. 1c). Exploring the entire $T M S$ database, $S h$ expression was largely confined to bladder epithelia (Extended Data 
Fig. 6a). Reviewing our recent work ${ }^{26}$, Ihh expression rose in the kidney at day 2 post-UUO (when fibrosis is initiated and leukocyte $\operatorname{Tnf}$ production maximal) and fell alongside Acta2 and Col3al with reversed UUO when leukocyte Tnf signalling and fibrosis are known to be reduced $^{26}$ (Extended Data Fig. 7a-c). This finding supported a role for $I h h$ as an early profibrotic ligand and was consistent with clinical studies in bone disease identifying $I h h$ as a $\mathrm{TNF} \alpha$ target ${ }^{27} . I S H$ in uninjured young and aged murine kidneys demonstrated $U b d$ and $I h h$ co-localisation within aged proximal tubules (Extended Data Fig. 7c).

To confirm the effect of IHH protein on fibrosis, we exposed cultured human renal fibroblasts to recombinant IHH or Hedgehog-agonist SAG (Smoothened Agonist, Extended Data Fig. 7d). IHH administration induced a $>10$ fold increase in COL1A1 transcript levels and increased ACTA2, FAP and TGFBR1 levels, with a similar pattern of induction seen with SAG (Extended Data Fig. 7e).

qPCR performed on kidneys from our murine IRI and UUO disease models demonstrated that Ihh and Collal transcripts were inhibited by TNF $\alpha$ antagonism with Infliximab vs vehicle, whilst $T g f b 1$ remained unchanged (Fig. 2e). To determine whether NFkB activation was necessary for TNF $\alpha$-induced $I h h$ synthesis and fibrosis, mice underwent IRI with post-injury

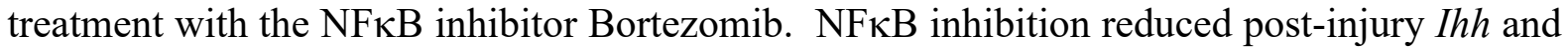
collagen deposition at transcript and protein level (Fig 2f).

\section{Renal epithelial Ihh production drives kidney fibrosis in two models of renal injury}

To determine the role of renal epithelial Ihh production in post-injury fibrosis we generated transgenic mice with selective and conditional genetic inhibition of renal epithelial Ihh signalling (with preserved $U b d, T g f b$, and $S h h$ gene function). Given the high expression of the 
renal epithelial restricted Pax 8 transcription factor Pax 8 in iPT cells, Ihh ${ }^{\mathrm{fl} / \mathrm{fl}}$ and Pax $8^{\text {creERT2 }}$ transgenic mice were crossed resulting in Pax $8^{\text {creERT2}} ; \mathrm{Ihh}^{\mathrm{fl} / \mathrm{fl}}$ mice with the ability to induce conditional Ihh deletion in renal epithelia by Tamoxifen administration (Fig. 3a, confirmed by ISH). This was necessary as constitutive $I h h$ deletion is lethal - in part due to renal agenesis. Tamoxifen treated Pax $8^{\text {creERT2}}{ }^{\text {Ihh }}{ }^{\mathrm{fl} / \mathrm{fl}}$ mice developed less fibrosis at $\mathrm{d} 7$ post UUO vs vehicle treatment (Fig. 3b-c). To control for tamoxifen induced changes, fibrosis was measured in tamoxifen treated transgenic and non-transgenic mice, showing protection with $I h h$ deletion and confirming the role of renal epithelial $I h h$ in promoting fibrosis.

IRI was induced in a single kidney in Pax $8^{\mathrm{creERT} 2}$; $\mathrm{Ihh}^{\mathrm{fl} / \mathrm{fl}}$ mice and non-transgenic littermates. Mice received tamoxifen 2 weeks pre and 1 week post IRI, with control transgenic animals receiving IRI + vehicle alone or no surgery + vehicle (Fig. 3d). Measurement of kidney weight and fibrosis demonstrated increased renal size and reduced fibrosis in kidneys with epithelial Ihh depletion (Fig. 3e). Overrepresentation analysis of RNA-Seq data demonstrated reductions in multiple fibrosis pathways including Hedgehog signalling in epithelial $I h h$ depleted kidneys compared to wild-type (Fig. 3f).

\section{$\mathrm{IHH}^{+} \mathrm{UBD}^{+}$iPT cells are upregulated in human ageing and kidney fibrosis}

To establish if $I H H^{+} U B D^{+}$iPT cells also exist in humans we undertook $I S H$ for IHH and UBD expression in human kidney tissue from young and aged kidneys pre-transplantation, and from a human kidney biopsy with progressive IgA nephropathy. In both a 'healthy' aged kidney and IgA nephropathy renal epithelia co-expressing IHH and UBD were identified in human kidney ageing and disease but not in young non diseased control kidney (Fig. 3g, Extended Data Fig. 8a). 


\section{Circulating IHH levels are higher in patients with progressive CKD}

We next tested whether patients with renal pathologies considered 'high risk' for progressive fibrosis in the kidney and heart (IgA and diabetic nephropathy) had higher levels of circulating IHH protein than patients with 'low risk' renal diagnoses including those associated with proteinuria (Minimal Change glomerulonephritis) and functional loss not usually associated with progressive fibrosis (unilateral nephrectomy). Patients with 'high risk' CKD had serum IHH levels 3x higher than those with 'low risk' disease, and lost function 5x more quickly over the next $>2.5$ years (Fig. $3 h$ ).

\section{Canonical Hedgehog signalling is profibrotic in experimental renal disease}

To clarify whether activation of canonical Hedgehog signalling through Smoothened induces fibrosis in the absence of other profibrotic stimuli in vivo, young healthy mice were dosed for 7 days with Smoothened Agonist (SAG, Fig. 4a). Treatment with SAG induced collagen deposition in the kidney and heart (Fig. 4b). Thus, canonical Hedgehog signalling activation is sufficient to drive fibrosis in multiple organ systems.

\section{Inhibition of canonical Hedgehog signalling reduces local and systemic fibrosis}

The impact of Cyclopamine mediated Smoothened inhibition (blocking canonical and noncanonical Hh signalling) and GANT61 mediated Gli1/2 inhibition (blocking canonical Hh signalling only) on fibrosis were compared, testing whether existing studies using Gli1/2 inhibition in experimental UUO could be extended to post-IRI fibrosis ${ }^{28}$. The impact of pharmacological Hh inhibition on proliferation of Gli1+ mesenchymal progenitor cells was tested using Gli1 ${ }^{\text {creERT2}}$;Ai14 $\left(\right.$ TdTom $^{\text {LSL }}$ ) mice pre-dosed with tamoxifen to label Gli1+ progenitor cells which are known to proliferate in response to injury and contribute to organ fibrosis $^{29}$ (Fig. 4c). 
Renal fibrosis was reduced following unilateral IRI with Cyclopamine and GANT61 treatment

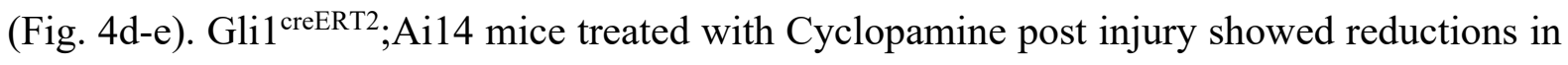
numbers of TdTomato+ cells reflecting cells derived from Glil expressing progenitors (Fig. 4f). GANT61 treated kidneys had reduced expression of Collal, Col3al and Tgf (Fig. 4g). Collectively, these results demonstrate that genetic $I h h$ depletion results in an equivalent reduction in fibrosis to pharmacological inhibition of pan-Hh signalling, a finding consistent with IHH being the major Hedgehog ligand mediating fibrosis in vivo.

\section{IHH is a non-redundant mediator of TNF $\alpha$ signalling induced fibrosis post-injury}

The ability of TNF $\alpha / \mathrm{IHH}$ signalling inhibition to protect kidney function and inhibit fibrosis when administered after established bilateral renal injury was tested. To do this, vehicle treated mice were compared to mice receiving either TNF $\alpha$ inhibition or canonical Hh inhibition starting $24 \mathrm{~h}$ post injury. The ability of TNF $\alpha$ inhibition to augment the renal protection seen with genetic ablation of epithelial Ihh expression was also tested (Fig 4h). Pharmacological inhibitors of TNF $\alpha / \mathrm{IHH}$ signalling reduced kidney fibrosis and preserved kidney function to a level comparable to epithelial Ihh knockdown (Fig. 4i-j). Of note, the addition of TNF $\alpha$ blockade to animals already lacking epithelial Ihh elicited no additional protection from fibrosis or functional improvement, consistent with a non-redundant role for IHH in TNF $\alpha$ profibrotic signalling.

\section{Renal injury induces transcriptional changes in remote organs}

Given that chronic kidney disease can progress even after an apparently discrete and resolved renal injury ${ }^{30}$ we tested whether the presence of a previously injured kidney resulted in long term effects on a distant uninjured contralateral kidney. Bulk RNAseq analysis demonstrated 
that in the presence of a fibrotic kidney injured 10 weeks previously, the uninjured kidney also downregulated inhibitors of inflammatory signalling and upregulated collagen transcript levels, compared to a mouse with a single uninjured kidney 10 weeks after a contralateral nephrectomy. Thus, previous renal injury mediates systemic effects independent of renal functional loss (Extended Data Fig. 9).

Hypertension and reduced renal clearance of waste products have both been proposed to contribute to the increased incidence of cardiac fibrosis seen even in early experimental and human $\mathrm{CKD}^{31-33}$. Returning to our model of transgenic Ihh depletion confined to Pax8 expressing epithelia with maintained cardiac Ihh expression (due to negligible cardiac Pax8 expression left unaltered by Tamoxifen, Online Data and Extended Data Fig. 10a) we analysed whether IRI to a single kidney induced cardiac fibrosis without advanced renal impairment (due to the presence of an uninjured contralateral kidney). Blood pressure readings were taken before, 1 week after and 10 weeks after injury pre-cull showing that no group had hypertension (Fig. 5a, Extended Data Fig. 10b). Measurement of cardiac left ventricular wall thickness and fibrosis levels demonstrated cardiac hypertrophy and fibrosis occurred in the absence of hypertension or advanced renal impairment and was reduced in the absence of Pax $8+$ epithelial Ihh production (Fig. 5b). Whilst unexpected, this finding was compatible with the published reports of increased Hedgehog signalling promoting cardiomyocyte and fibroblast proliferation within the heart independent of blood pressure changes ${ }^{34,35}$. Echocardiography of a subset $(n=4)$ of each group demonstrated preserved cardiac function, with significant LV mass increase in Ihh WT mice post IRI compared to unilateral nephrectomy only, which was not present in $\mathrm{Pax} 8^{\mathrm{creERT}}$; $\mathrm{Ihh}^{\mathrm{fl} / \mathrm{fl}}$ mice post IRI. Interrogating the $T M S$ database, we detected Hh activation in cardiac fibroblasts, despite minimal $I h h$ and absent $S h h$ transcript production in any cardiac cell type (Extended Data Fig. 10a). These findings support the ability of circulating 
hedgehog ligands to induce remote fibrosis, consistent with recent reports of raised systemic Hh signalling in human patients with renal failure ${ }^{36}$.

\section{Pharmacological inhibition of Hh signalling inhibits remote fibrosis after kidney injury}

To determine whether downstream pharmacological inhibition of $\mathrm{Hh}$ signalling would recapitulate the effects of genetic IHH depletion upon fibrosis in remote organs we pooled results from all studies of pharmacological Hedgehog signalling inhibition in IRI, allowing differences in fibrosis seen in uninjured contralateral kidneys and hearts to be compared (Fig. 5c). At 6 weeks post-IRI fibrosis was increased in contralateral kidneys and hearts of vehicle treated post-IRI animals compared to animals undergoing identical injury with post-injury Hedgehog inhibition (Fig. 5d-e). To control for the impact of IRI on renal function, uninjured animals were compared to animals which had undergone unilateral nephrectomy - inducing an equal/larger functional decrease without post-injury iPT cells. Post-IRI animals had the highest levels of fibrosis in hearts and uninjured kidneys, with pharmacological inhibition of Hedgehog signalling blocking fibrosis in both organs (Fig. 5d-e). 


\section{Discussion}

Chronic kidney disease becomes more common with age, affects hundreds of millions of people worldwide and is a major risk factor for accelerated cardiovascular disease and premature death ${ }^{37,38}$. Chronic inflammation is a feature common to all advancing kidney disease, but the mechanism underlying any connection remains incompletely understood. This work addressed the hypothesis that changes in the renal epithelial phenotype linked aging and inflammation to systemic fibrosis.

In this study, we explored ageing and injured human kidneys at single cell resolution, identifying a novel renal epithelial cell subset which produced Ihh in response to TNF $\alpha$ exposure. Using human cell culture, murine experimental kidney disease and human kidney biopsies we demonstrate that IHH is a major driver of fibrosis in the kidney and heart, with inhibition of TNF $\alpha, \mathrm{NF} \kappa \mathrm{B}$ or Hedgehog signalling blocking these effects in both organs after injury (summarised in Figure 6). These data link the immune activation known to be present in ageing, inflammation and organ injury ${ }^{3-5}$ to the predisposition towards progressive fibrosis seen in all these conditions ${ }^{8,9}$.

Our findings of reduced cardiac hypertrophy and fibrosis with blockade of the Hedgehog pathway downstream of TNF $\alpha$ are consistent with published work showing that TNF $\alpha$ knockout mice are protected from cardiac hypertrophy ${ }^{39}$. Existing work in the heart has focused on the beneficial impact of Sonic Hedgehog administration on cardiomyocyte and endothelial proliferation in the immediate aftermath of ischaemic injury ${ }^{35}$. This has led to interest in the use of Hedgehog agonism to promote myocardial viability after injury ${ }^{40}$. Our data raises the possibility that prolonged agonism or raised levels of circulating hedgehog 
ligand may worsen cardiac fibrosis and induce ventricular hypertrophy - both features associated with reduced survival in patients with $\mathrm{CKD}^{8}$. It is noteworthy that serum levels of IHH were significantly higher in patients with progressive CKD at high risk of cardiovascular events. Our results are also consistent with the reported impact of Hedgehog signalling on cardiac fibroblast proliferation ${ }^{35}$, where acute $\mathrm{SHH}$ administration led to expanded alpha-SMA positive fibroblast staining.

Our data provides a mechanism linking kidney inflammation to kidney fibrosis. Whilst published work supports the importance of downstream Hedgehog signalling in renal fibroblasts in rodent models of $\mathrm{CKD}^{25}$, this has been reported to reflect the widespread induction of Sonic Hedgehog protein in tubular epithelial cells after injury ${ }^{25,41}$. Using our own scRNAseq datasets we did not see comparable upregulation of Shh after experimental renal injury, and the profound reduction in collagen deposition seen in animals lacking Ihh but with preserved Shh gene function supports a key and previously underappreciated role of $I h h$ as a fibrotic mediator.

The current study has limitations. No murine model can fully replicate the multiple pathologies contributing to human kidney disease. Our studies focused on monitoring response to the injured kidney in vivo, and whilst histology and echocardiography showed evidence of cardiac hypertrophy and fibrosis there was no major cardiac dysfunction seen in the timeframes studied here. Further studies of experimental cardiac disease and larger, prospective studies in human disease will be required to validate and extend the work presented here.

Whilst our scRNAseq dataset indicates that TNFa upregulation in the kidney after injury reflects increased leukocyte production rather than increased expression from the renal 
parenchyma, in the future it would be of value to explore the impact of systemic vs leukocyte restricted TNF $\alpha$ deficiency to determine if any role for low level non-leukocyte TNF $\alpha$ production exists. Other groups have identified distinct populations of $\mathrm{Vcaml}^{+}$and $\mathrm{Sox} 9^{+}$ epithelial cells in the kidney either at baseline ${ }^{19}$ or in the early aftermath of injury ${ }^{42}$ - reported to be "injured" or "regenerating", and associated with upregulated NFkB ${ }^{19}$. $U b d+\mathrm{iPT}$ cells have upregulated expression of both Vcam 1 and $\operatorname{Sox} 9$ in baseline aged and fibrotic kidneys, and the transcriptional responses to TNF $\alpha$ blockade would be consistent with all these markers identifying a single population of TNF $\alpha$ activated epithelia. Precedents exist in other organ systems for this, with TNF $\alpha$ administration inducing VCAM1 expression via NFkB activation in human pulmonary epithelial cells in vitro ${ }^{43}$.

Our findings raise questions about the long-term consequences of Hedgehog signalling induction systemically and in the heart. It will be important to conduct further studies exploring genetically targeted and pharmacological inhibition and activation of single gene and panHedgehog signalling, monitoring their impact on cardiac and renal function. Experimental models exploring acute and chronic myocardial injury and interrogation of existing clinical datasets using inhibitors of the pathays in question will be of great value in determining optimal levels of TNFa/hedgehog activity for short and long term renal and cardiac health. Encouragingly, in rheumatoid arthritis (RA), treatment with biologic agents ( $>90 \%$ of which were TNF $\alpha$ antagonists) reduced the incidence of CKD with eGFR $<45 \mathrm{ml} / \mathrm{min}$ by $29 \%{ }^{44}$ whilst TNF $\alpha$ antagonists stabilised renal function in patients with RA and CKD compared to other disease modifying drugs ${ }^{45}$. TNF $\alpha$ antagonist treatment reduced the incidence of total cardiovascular events compared to other agents in RA patients, with protection increasing with longer treatment duration ${ }^{46}$. Given their well-established side effect profile and efficacy, a trial 
of the impact of TNF $\alpha$ antagonism on renal functional decline and cardiovascular outcomes in patients with progressive $\mathrm{CKD}$ would represent a logical progression from this work.

Our data demonstrate that IHH release from iPT cells in the kidney links inflammatory $\mathrm{TNF} \alpha$ release to multi-organ fibrosis and represents a deleterious arm of the ageing and injuryresponse. TNF $\alpha, \mathrm{NF} \kappa \mathrm{B}$ and Hedgehog antagonists are licenced for clinical use for other disorders, and their efficacy as treatments for progressive cardiorenal fibrosis in aging, in CKD, after renal transplantation and as prophylaxis around acute illness in aged and vulnerable patients warrants further investigation. 


\section{Materials and Methods}

\section{Study Design}

This study addressed the hypothesis that changes in the renal epithelial phenotype linked ageing and inflammation to systemic fibrosis. To answer this a series of controlled laboratory experiments were performed with the primary objective of determining whether distinct subsets of renal epithelia could be identified from models of renal injury and ageing, and their contribution to renal fibrosis defined. All studies were conducted in accordance with ARRIVE guidelines ${ }^{47}$. Based on previous work a two-sided two sample test was used to generate group sizes for all studies. All studies were performed at the Harvard Medical School, Boston, MA, USA and the University of Edinburgh, UK, with data collection performed at pre-specified endpoints unless limited by animal distress as identified by facility staff and/or veterinary surgeons. No available data was excluded from the analysis. Primary and secondary endpoints were pre-specified. Each mouse represented one experimental unit. In all experiments, there was randomised allocation to groups. Drug administration and surgical timing was rigorously rotated across all groups. Tissue was collected by two staff members blinded to the treatment groups, using a long established, validated coding system for each animal. Semi-blinded staining and image analysis was undertaken with unblinding only being performed after all data acquisition was complete.

\section{Human biopsies}

After appropriate ethical approval from Tissue Governance at the Royal Infirmary of Edinburgh, and under the remit of the UK QUOD Consortium supported by NHS Blood and Transplant (REC reference number 20/SS/0105) sections were obtained from human renal biopsy tissue from native renal disease and pre-implantation renal transplants. 


\section{Cell culture}

Human renal proximal tubular epithelial cells (hRPTEC; ATCC) were maintained in DMEMF-12 + Glutamax-1 supplemented with hTERT immortalised RPTEC growth kit (ATCC) and $50 \mathrm{mg} / \mathrm{mL}$ Geneticin (Gibco-Life Technologies, USA). Cells were grown at $37^{\circ} \mathrm{C}$ in $5 \% \mathrm{CO}_{2}$. hRPTEC cells were plated at $2 \times 10^{5}$ cells per well of a 6 well plastic culture dish. Cells were incubated for 7 days with media change every 3-4 days. $T N F \alpha / N F \kappa B$ inhibition: Cells were grown in serum free media for 48hrs, incubated for 1hr with BAY 11-7082 (or vehicle), with recombinant TNFa (Sigma, USA) added for $24 \mathrm{hrs}$ at $20 \mathrm{ng} / \mathrm{mL}$. Human primary kidney fibroblasts (H-6016, Cell biologics, Chicago, IL, USA) were plated at $3 \times 10^{6}$ cells per well in a 6 well plastic culture dish plate pre-coated with gelatin based coating solution (6950, Cell biologics). Cells were maintained in complete fibroblast medium (M2267, Cell biologics). Cells were grown at $37^{\circ} \mathrm{C}$ in $5 \% \mathrm{CO}_{2} .24$ hours before treatment with protein of interest, cells were washed once with PBS and media was swapped for serum free media (M2267SF, Cell Biologics). Hedgehog activation: Cells were then treated with Smoothened Agonist (SAG) at $1.25 \mathrm{nM}$ (MedChemExpress, NJ, USA) or IHH at $5 \mu \mathrm{g} / \mathrm{mL}$ (Source Bioscience, UK) in serum free media. 72 hours later cells were collected for qPCR.

\section{Experimental mice}

All mice were on either a C57BL/6 or a mixed genetic background. Ihh fl/fl, Ai14 reporter and Gli1-cre/ERT2 mice (Stock nos 024327, 007914 and 007913) were purchased from the Jackson Laboratory (MI, USA). Pax8-cre/ERT2 mice (Stock no 10364) were purchased from the European Mutant Mouse Archive (Munich, Germany). All experiments comprised male mice, unless otherwise stated. Ages ranged from 6-8 weeks for young and 18-24 months for old. Mice were bred and maintained at the University of Edinburgh, UK and Harvard Medical School, 
Boston, USA, obtained from the National Institute on Ageing, Bethseda, MD, USA or purchased from Harlan, UK. All animal work was compliant with IACUC guidelines, conducted in accordance with the UK Government Animals (Scientific Procedures) Act 1986, in conventional barrier unit facilities with conventional bedding, $12: 12 \mathrm{~h}$ light:dark cycle, ambient temperature control and access to food and water ad libitum and was approved by the University of Edinburgh Ethical Review Committee or the IACUC, Harvard Medical School, Boston, MA, USA.

\section{Ischaemia Reperfusion Injury (IRI) and Unilateral Ureteric Obstruction (UUO)}

IRI surgery was performed as previously described ${ }^{48}$. Anesthesia was induced with $2 \%$ isofluorane. Buprenorphine analgesia was administered subcutaneously. A flank incision was performed and either one or both renal pedicles identified and clipped using an atraumatic clamp. During the ischemic period, body temperature was maintained using a heating blanket with homeostatic control (Harvard Apparatus, USA) via a rectal temperature probe. After clamp removal, peritoneal closure with 5/0 suture and skin closure with clips was performed prior to $\mathrm{s} / \mathrm{c}$ administration of $1 \mathrm{~mL} 0.9 \%$ saline. In experiments using unilateral IRI the right kidney was always uninjured and is referred to as the contralateral kidney (CLK). Animals were maintained for up to 10 weeks post-IRI before tissue harvest, and non-invasive blood pressure monitoring performed. Kidneys were weighed at time of sacrifice.

UUO surgery was performed as previously described ${ }^{49}$. Anesthesia was induced with $2 \%$ isofluorane. Buprenorphine analgesia was administered subcutaneously. A flank incision was performed and the left ureter identified and ligated using a vascular clip (HemoClip Plus, Weck, USA). After UUO surgery animals were maintained for up to 7 days before tissue 
harvest. Tissue was fixed in Methcarn (Methanol 60\%, Chloroform 30\%, Glacial Acetic Acid $10 \%), 10 \%$ Formalin and $4 \%$ Paraformaldehyde or snap frozen in liquid nitrogen/dry ice.

\section{In vivo pharmacological interventions}

Tamoxifen administration: Tamoxifen (Sigma, USA) was dissolved at $40 \mathrm{mg} / \mathrm{mL}$ in corn oil and administered by gavage at $200 \mathrm{mg} / \mathrm{kg}$ for three doses on alternate days. TNF $\alpha$ inhibition: Infliximab (Selleckchem, USA) was reconstituted in $0.9 \%$ saline and administered once only at a dose of $10 \mathrm{mg} / \mathrm{kg}$ i.p. NFאB inhibition: Bortezomib (Selleckchem, USA) was diluted in 2\% DMSO, 30\% PEG300 and distilled water and administered by gavage three times weekly at $1 \mathrm{mg} / \mathrm{kg}$. Hedgehog pathway agonism: SAG (MedChemExpress, NJ, USA) was dissolved in dimethyl sulfoxide (DMSO) to $5 \mathrm{mM}$ and further diluted with normal saline prior to daily i.p injection at $20 \mu \mathrm{g} / \mathrm{g}$. Hedgehog pathway inhibition: Cyclopamine was complexed with $45 \% 2$ hydroxypropyl- $\beta$-cyclodextrin (HBC, Sigma, USA) and sterile PBS and gavaged daily at a total dose of 5mg/kg. GANT61(MedChemExpress, NJ, USA) was dissolved in 20\% ethanol and $80 \%$ corn oil, and administered by gavage at $50 \mathrm{mg} / \mathrm{kg}$ on alternate days. In all cases, volume matched vehicle was given to controls.

\section{Immunohistochemistry (IHC)}

Picrosirius red: For picrosirius red (PSR) staining, sections were deparaffinised and rehydrated before treatment in haematoxylin for 8 minutes. After washing, these were stained in picrosirius red for 1 hour, before acidified water washing, dehydration and mounting. H\&E: sections were deparaffinised and rehydrated before staining with haematoxylin and eosin, before acidified water washing, dehydration and mounting. 
Labelling of RNA transcripts in paraffin embedded tissue was performed using the RNAScope2 platform (ACD-Bio, USA). Probes to Ubd, Collal and Ihh were applied and visualised as previously described ${ }^{50}$.

\section{Microscopy}

For studies of in vitro cultures random fields of view within the plate (at x20 magnification) were captured using light microscopy on an Evos microscope. Scaling was performed using a graticule. For each well, multiple random fields of view were captured for analysis and the results averaged prior to cell lysis in trizol for RNA extraction. For IHC and FISH kidney sections were visualized and captured using the Zeiss Axioskop 2mot+ or on an Axioscan Z1 slidescanner. Confocal imaging was performed on a Leica SP8 Confocal Microscope (Leica, Germany). In all cases image analysis was performed using Image $2.1(\mathrm{NIH}$, USA) or in QuPath 0.2.3 ${ }^{51}$.

\section{$R N A$ extraction and $q P C R$}

RNA was extracted from human PTECS or murine kidneys using TRIZOL (Invitrogen, Carlsbad, CA) according to the manufacturer's instructions and reverse transcribed to cDNA (Qiagen Quantitect Reverse Transcription Kit). qPCR was carried out using Perfecta qPCR mastermix (VWR, UK) with TAQman ${ }^{\circledR}$ gene expression assays (ThermoFisher, Waltham, Massachusetts, U.S.A) on a Roche Lightcycler 480 using standard protocol.

For human RPTEC cells, the TAQman ${ }^{\circledR}$ gene expression assays used were glyceraldehyde 3phosphate dehydrogenase (Hs02758991_g1 GADPH), hypoxanthine-guanine phosphorribosyltransferase (Hs02800695 HPRT1), peptidylprolyl isomerase A (Hs04194521 PPIA), tumour necrosis factor (Hs00174128_m1 TNF), transforming growth factor (Hs00998133_m1 
TGFB1), Ubiquitin D (Hs00197374_m1 UBD), collagen 1a1 (Hs00164004_m1, COL1A1), Alpha-smooth muscle actin (Hs00426835_g1, ACTA2), Fibroblast activation protein (Hs00990813_m1, FAP) and TGF-beta receptor 1 (Hs00610322_m1, TGFBR1). mRNA expression was normalized for GAPDH, HPRT1 and PPIA (average) expression and presented as fold increases over naive.

For murine kidneys, the following TAQman ${ }^{\circledR}$ gene expression assays were used: House keeping genes: hypoxanthine-guanine phosphoribosyltransferase (Mm03024075_m1 Hprt), Gapdh (Mm99999915_g Gapdh), Peptidylprolyl Isomerase A (Mm0234230 PPia) Phosphoglycerate kinase 1(Mm00435617_m1 Pgk1); transforming growth factor (Tgf; Mm01178820_m1 Tgfb1), Fibrosis markers: collagen 1a1 (Mm00801666_g1 Colla1), $\alpha-$ smooth muscle actin (Mm00725412_s1 Acta2). iPT markers: Indian Hedgehog (Hs00745531_s1), Ubiquitin D (Mm01972246_s1, Ubd). mRNA expression was normalized for HPRT or average HK expression and presented as fold increases over age-matched control analysed in parallel.

\section{Kidney digestion for sequencing.}

Immediately after culling, mice were perfused with $10 \mathrm{~mL}$ PBS. Kidneys were excised, decapsulated and placed in ice-cold PBS. Equal portions of renal cortex from each mouse were finely minced in digest buffer $[4.25 \mathrm{mg} / \mathrm{mL}$ Collagenase V (Sigma-Aldrich St. Louis, Missouri, USA), $6.25 \mathrm{mg} / \mathrm{mL}$ Collagenase D (Roche, Basel, Switzerland), 10mg/mL Dispase (Thermo-

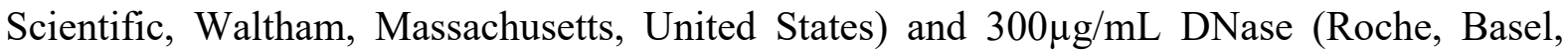
Switzerland) in RPMI 1640 (10\% fetal calf serum, 1\% penicillin/streptomycin/L-glutamine)] prior to homogenisation in gentleMACS C-tubes using the gentleMACS dissociator (Miltenyi Biotec, Auburn, California, USA). Samples were incubated at $37^{\circ} \mathrm{C}$ with shaking to maximise 
digestion. The kidney suspension was then subjected to a second gentleMACS homogenisation and digestion neutralised with an equal volume of FACS buffer (PBS, 2mM EDTA and 2\% FCS). Kidney cell suspensions were then passed sequentially through $100 \mu \mathrm{m}, 70 \mu \mathrm{m}$ and 40 $\mu \mathrm{m}$ sieves. Any residual red blood cells were lysed by RBC lysis buffer (Sigma-Aldrich St. Louis, Missouri, USA). Cells were re-suspended in ice-cold FACS buffer ready for use.

\section{Single-Cell droplet library preparation}

For single-cell RNA-Seq analysis on the 10x Genomic platform (10x Genomics, Pleasanton, California, USA), single-cell suspensions from renal cortex were prepared from pools of 3 animals from each group, as outlined by the 10x Genomics Single Cell 3' v2 Reagent kit user guide. 50,000 live (DAPI-) cells were sorted on the BD FACS ARIA II. Samples were washed twice in PBS followed by centrifugation at $500 \mathrm{~g}$ for 5 minutes at $4^{\circ} \mathrm{C}$. Sample viability was assessed using trypan blue with an automated cell counter (Bio-Rad, Hercules, California, USA) and the appropriate volume for each sample was calculated. The chip was loaded with 10700 cells per lane. After droplet generation, samples were transferred onto a pre-chilled 96well plate, heat-sealed and reverse transcription was performed using a c1000 touch thermal cycler (Bio-Rad, Hercules, California, USA). After reverse transcription, cDNA was recovered using the 10x Genomics Recovery Agent and a Silane DynaBead cleanup (ThermoFisher, Waltham, Massachusetts, U.S.A) was performed. Purified cDNA was amplified and cleaned using SPRIselect beads (Beckman, Brea, California, U.S.A). Samples were diluted at 4:1 (elution buffer (Qiagen, Düsseldorf, Germany)/cDNA) and an initial concentration check performed on a Qubit fluorometer (Invitrogen/ThermoFisher, Waltham, Massachusetts, U.S.A) to ensure adequate cDNA concentration. Final cDNA concentration was checked on a bioanalyzer (Invitrogen/ThermoFisher, Waltham, Massachusetts, U.S.A). 


\section{Single cell RNA-Seq}

We generated libraries ( 3 biological replicates per library) from uninjured mice and mice 42 days following IRI surgery as described previously. These single cell libraries were generated from whole kidney digests and prepared using a high-throughput droplet-based library preparation workflow (10x v2). The sample was then split and sequenced across 4 lanes on a single Illumina flow cell on a NextSeq 550 High Output Kit v2 (Illumina) for 150 cycles at $400 \mathrm{M} P \mathrm{PE}$ reads comprising of 2x75bp and $8 \mathrm{bp}$ index reads.

The Tabula Muris Senis data follows a similar droplet based protocol using the 10x platform with digests from mice of multiple ages and is described in detail in the original manuscript ${ }^{15}$.

\section{Single-cell RNA sequencing analysis}

The cellranger mkfastq wrapper (Cell Ranger Single Cell software suite 2.1.0, http://10xgenomics.com) de-multiplexed the illumina output BCL files to library specific FASTQ files. Subsequently, alignment was performed using the cellranger count function using STAR aligned 2.5.1b against the Ensembl mouse reference genome version GRCm $38.68^{52}$. Correction and filtering of cell barcode and unique molecular identifiers followed, and the retained barcodes were quantified and used to generate a gene expression matrix.

A similar workflow was used for the IRI and TMS dataset, following a standard sequence of procedures of filtering, highly variable gene selection, dimensionality reduction and clustering were performed using the single cell RNAseq analysis R package Seurat(v3) $)^{53}$. 
Following alignment and pre-processing, we filtered out any features not present in at least 3 cells and any cells without at least 200 features. To exclude low-quality cells in both singlecell experiments, we then filtered cells that expressed fewer than 300 genes per 500 unique molecular identifiers, and to exclude probable doublets, cells with $>10,000$ unique molecular identifiers and $<3,000$ genes were removed. This will have removed the majority of injured and apoptotic cells ${ }^{54}$. We used a mitochondrial filter to remove cells in which $>50 \%$ of genes were mitochondrial, consistent with other renal-specific scRNA-Seq projects. This is a higher filter than has been used in non-renal single-cell analysis but reflects the high mitochondrial content in renal tubular epithelial cells. In the case of the TMS data, the matrix was already processed as per the original manuscript prior to access so this step was skipped. Following this QC, we began our analysis workflow with 3063 cells with 15340 total features in the IRI data, 1313 cells with 13217 total features in the uninjured data 21647 cells with 20138 total features in the TMS dataset.

Normalisation was performed using the Seurat package to reduce biases introduced by technical variation, sequencing depth and capture efficiency. We employed the default globalscaling normalisation method "logNormalize" which normalised gene expression per cell by the total expression and multiplies the result by a scaling factor before log transformation. We then scaled the data and regressed out variation between cells due to the number of unique molecular identifiers and the percentage of mitochondrial genes.

The expression matrix subsequently underwent dimensionality reduction using principal component analysis (PCA) of the highly variable genes within the dataset. Using Seurat's FindVariableGenes function (and computed using the LogVMR argument) we used log-mean expression values between 0.0125 and 3 , and a dispersion cut-off of 0.5 to select genes. PCA 
was performed using these selected genes and 20 principal components were identified for subsequent analysis in each dataset, selected both visually using the elbow point on the elbowplot and via the Jackstraw method.

Clusters were then identified using Seurat's FindClusters function, built using the first 20 principal components and a resolution parameter of $0.5-1.5$. The original Louvain modularity optimization algorithm was employed. UMAP was then used for further dimensionality reduction and visualisation, which was run on a reduced dimensional space of the first 20 dimensions. Epithelial cell clusters were classified based on expression of known signature genes. This resulted in 11244 cells with 20138 total features (mean 2417 features per cell) in the TMS data, 1437 cells with 15340 total features (mean 1107 features per cell) in the IRI data and 1187 cells with 13217 features (mean 1085 features per cell) in the uninjured data. These clusters where then subsetted out, renormalized, scaled, clustered and classified as before.

For all single-cell differential expression tests, we used the Wilcoxon rank-sum test to identify a unique expression profile for each cluster, with differential expression tested between each cluster and all other clusters combined. The FindAllMarkers test as implemented in Seurat returns an "adj_pval” (Bonferroni adjusted p-values) and an "avg_logFC" (average log fold change) for each gene. Genes were ranked in order of average log fold change and visualised using heatmaps. In some places specific clusters are compared to each other, for example iPT was compared to PCT cells in isolation, as they were the most similar cells type and likely "cell of origin" from which the iPT, thus DEG would be most incisive and least likely to be obscured by regional variation in transporters etc along the tubule.

\section{Bulk RNA-Seq}


Prior to RNA sequencing, RNA integrity was checked using Genechip (ThermoFisher), with only samples with RIN $>7$ when rechecked by the sequencing facility used to generate libraries utilized in subsequent analysis. A PolyA library was constructed and run on an Illumina Novaseq using $5 \times 150 \mathrm{bp}$ paired-end sequencing at a depth of 50M reads per library by Genewiz (NJ, USA).

FastQC was used for initial quality control assessment before trimming was performed with Cutadapt. This followed the standard procedure of first trimming low quality base calls from the 3 ' end of the read, before adapter trimming using the default settings- using the first $13 \mathrm{bp}$ of Illumina standard adapters ('AGATCGGAAGAGC')). The "- -paired" argument was used which runs a paired-end validation on both trimmed _ 1 and _2 FastQ files once the trimming has completed. This step removes entire read pairs if at least one of the two sequences became shorter than a certain threshold.

Reads were then mapped to GRCm38.68 mouse reference transcriptome, mm10 (Ensembl 93) was used using pseudo-aligner with Salmon v1.4. A quantification table of the transcripts was generated from this step for subsequent differential expression analysis. Mean mapping rate was $45.2 \%(35.3-64.8)$.

DESeq2 requires raw counts to run on as it internally normalises during calculation. For initial QC related visualisations such as Euclidean distance matrices, a variance stabilizing transformation was applied to the count data.

Differential expression analysis was performed in the $\mathrm{R}$ environment using DESeq2. This workflow imports the transcript-level quantification data output by Salmon, aggregating to the 
gene-level with tximeta. Firstly, as counts for transcripts in RNA-Seq data can contain many rows with only zeros, rows with little to no information about the amount of gene expression were removed to reduce the size of the data object and to increase computational speed. We removed rows from the expression matrix which had no counts, or only a single count across all samples.

Initial QC was performed using PCA plots data having applied a variance stabilizing transformation, with the "blind" argument set to "True" (this is later set to "False" for downstream analysis). All statistical analyses were performed on raw counts, as required by DESeq2, and exploratory visualisations such as covariance matrixes and PCA were performed on transformed data following the variance Stabilizing Transformation (VST) implemented in DESeq2. This function estimates this dispersion trend by sub-setting on a small number of genes chosen deterministically, to span the range of genes' mean normalized count. DESeq2 feature counts and other functions were run on the tables of counts to determine differentially expressed genes between groups. Results were considered statistically significant at an adjusted $\mathrm{p}<0.05$.

\section{Pathway Analysis}

Over representation analysis (ORA) was used to identify enriched pathways based on the differential expressed genes using WebGestalt. The Benjamini-Hochberg method was used to correct for multiple testing during ORA, and the top ten enriched categories as ranked by false discovery rate were selected. The reference gene list used was the Illumina mouseref 8 . We used pathway gene sets from the protein analysis through evolutionary relationships, PANTHER, Reactome and Kyoto encyclopaedia of genes and genomes as our reference gene lists. 


\section{Data Availability}

Data was deposited in the National Center for Biotechnology Information Gene Expression Omnibus database (accession \# GSE178109, secure token for reviewer access pre-publication access is qlyhemowzrgbpyf). Data from Tabula Muris Senis can be found at https://tabulamuris-senis.ds.czbiohub.org/.

\section{Statistical Analysis}

Data were analysed with Excel (Microsoft, USA), Prism 9.0 (GraphPad, USA) or R version 4. Multivariable linear regression was performed on $\mathrm{R}$ using the finalfit package (1.0.2). All values are expressed as mean values with error bars representing standard deviations. For all in vivo studies n numbers reflect biological replicates. Depending on the results of normality testing groups were compared using unpaired Student's T-test, Mann-Whitney U-test, one/two-way ANOVA (Tukey post-hoc test) or Kruskal-Wallis tests. P-values $<0.05$ denote statistical significance, ${ }^{*} \mathrm{p}<0.05,{ }^{* *} \mathrm{p}<0.01,{ }^{* * *} \mathrm{p}<0.005, * * * * \mathrm{p}<0.001$. 


\section{Acknowledgements}

The authors gratefully acknowledge the staff from the Queen's Medical Research Institute and the Bioresearch and Veterinary services facility at Edinburgh University for their expertise \& assistance in this work and the assistance of Professor Bob Hill in obtaining Ihh $\mathrm{fl} / \mathrm{fl}$ mice. The authors alto thank Professors Stuart Forbes and Sir John Savill and Dr Prakash Ramachandran for their proof-reading and advice on this manuscript.

\section{Funding}

EOS was supported by a Clinical Training fellowship from Kidney Research UK [TF_006_20161125]. KJM is supported by a Chief Scientist Office/Kidney Research UK Fellowship [CSO_PDF/2018/1]. LD is supported by a Senior Fellowship from Kidney Research UK [SF_001_20181122]. DAF was supported by an Intermediate Clinical Fellowship from the Wellcome Trust [100171/Z/12/Z]. 


\section{Figure Legends}

\section{Figure 1. Identification of inflammatory proximal tubular cells in ageing and injured}

kidneys. a. Single cell analysis of fibrotic and aged kidneys. b. UMAP plots of aged and injured renal epithelia. c. Key cell identity, fibrosis and TNF-related Differentially expressed genes in epithelial cell clusters. d. Over-representation analysis of inflammatory proximal tubules. False discovery rate $<0.05)$.

a single cell sequencing of fibrotic kidneys post IRI

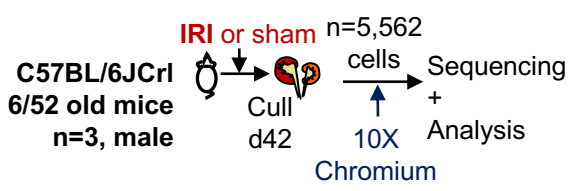

b UMAP plots of uninjured and fibrotic kidneys

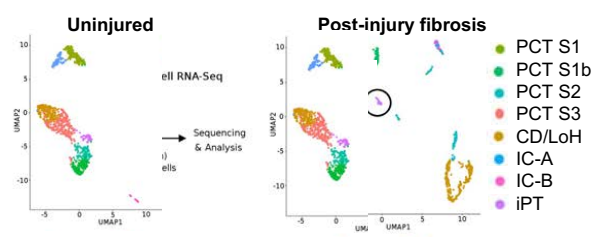

TMS kidney aging single cell sequencing studies

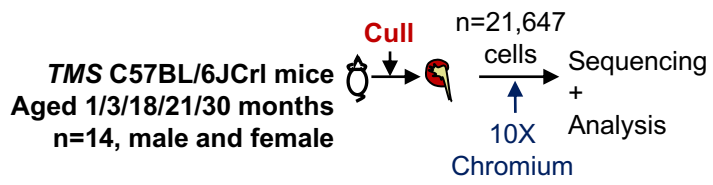

TMS Renal epithelial subsets with age distribution

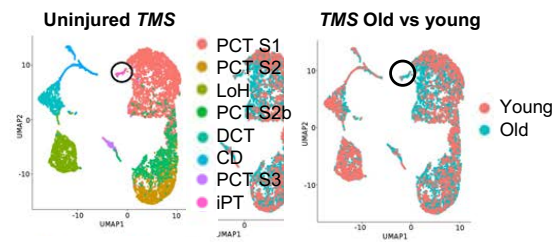

C Markers of iPT cells, fibrotic ligands and TNF $\alpha$ transcriptional targets in fibrotic and TMS kidneys
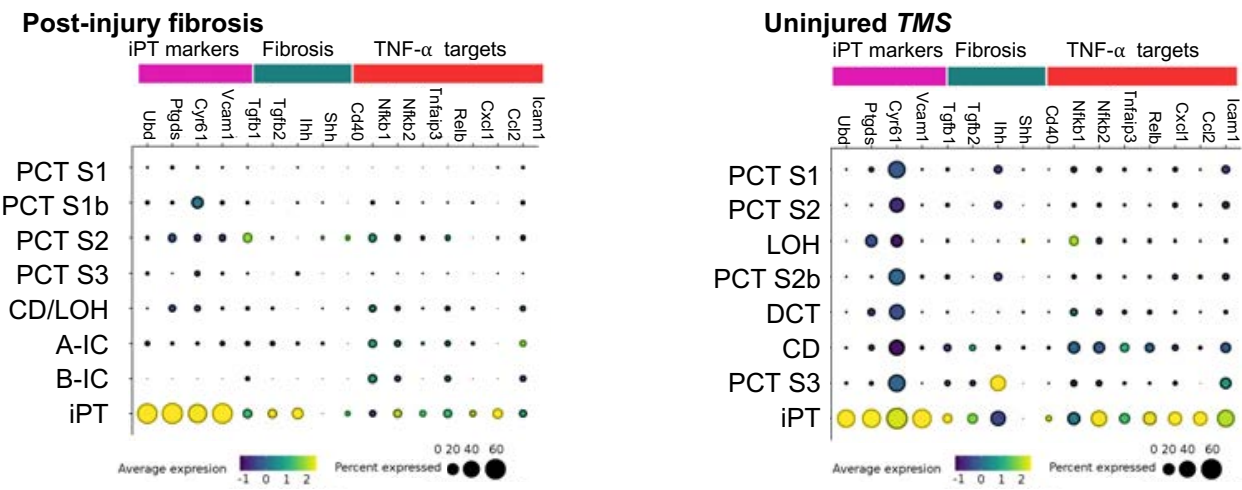

d Over representation analysis of IPT vs PCT cells in post IRI fibrosis and uninjured TMS kidneys

\section{Post-injury fibrosis} TNF signaling pathway
Primary Focal Segmental Glomerulosclerosis
MAPK signaling pathway

Dectin-1 mediated non-canonical NF-kB signaling $\mathrm{NIK} \rightarrow$ non-canonical NF-kB signaling TNFR2 non-canonical NF-kB pathway Cellular responses to stress
KEGG - Wikipathway - Reactome 
Figure 2. Profibrotic inflammatory proximal tubular cells are induced by TNF $\alpha$ in vivo. a. Schema of TNF $\alpha$ inhibition studies. b. TNF $\alpha$ inhibition reduces renal fibrosis after ischaemic injury. c. TNF $\alpha$ inhibition reduces renal fibrosis after obstructive injury in aged mice. d. Ubd+ iPT cells are induced by injury and reduced by TNF $\alpha$ inhibition. e. TNF $\alpha$ inhibition reduces Ihh and Collal transcript expression after injury. f. Inhibition of NFkB staining using Bortezomib inhibits $I h$ induction and collagen expression at a transcript and protein level in the murine kidney. Scale bars: 100microns (b,f), 50microns (c), 20microns (d). b-f: $n=6$ 14/group. f: $\mathrm{n}=74 .{ }^{*} \mathrm{p}<0.05,{ }^{*} \mathrm{p}<0.01, * * * \mathrm{p}<0.001, * * * * \mathrm{p}<0.0001$. Students t-test (b-c), one way ANOVA (d-f). 
a TNF $\alpha$ blockade Experimental Schema b

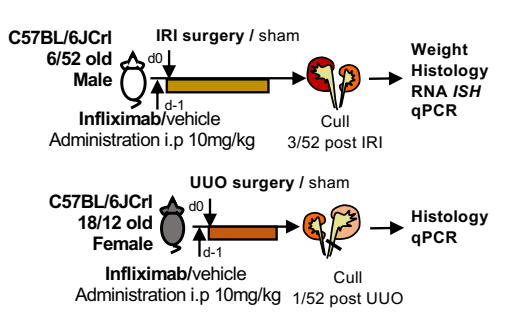

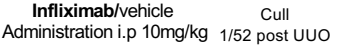
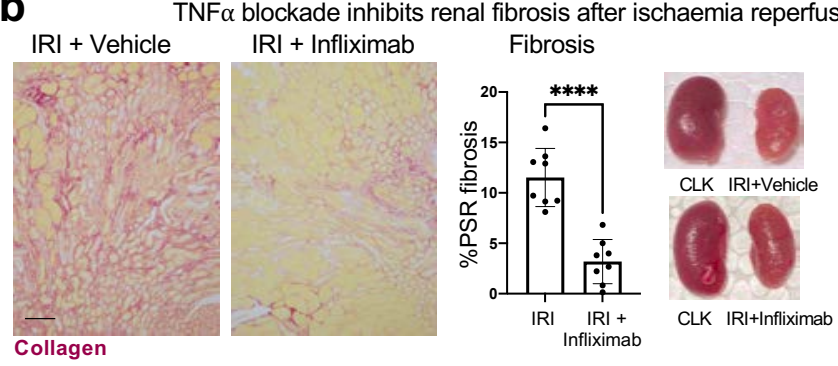

ion injury Injured/Healthy mass

C TNF $\alpha$ blockade inhibits renal fibrosis after UUO in vivo $\mathrm{UUO}+$ Vehicle $\quad \mathrm{UUO}+$ Infliximab $\quad$ Fibrosis

d Ubd+ iPT cells are induced in the aftermath of renal injury and inhibited by TNF $\alpha$ blockade
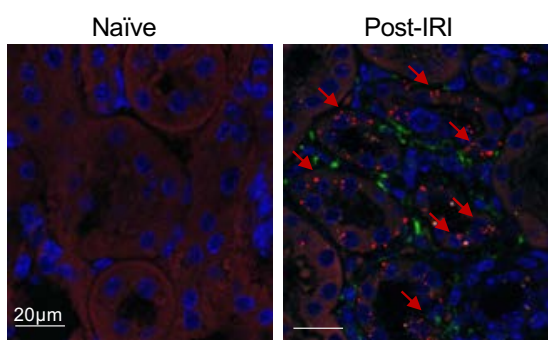

Post-IRI +anti-TNF $\alpha$
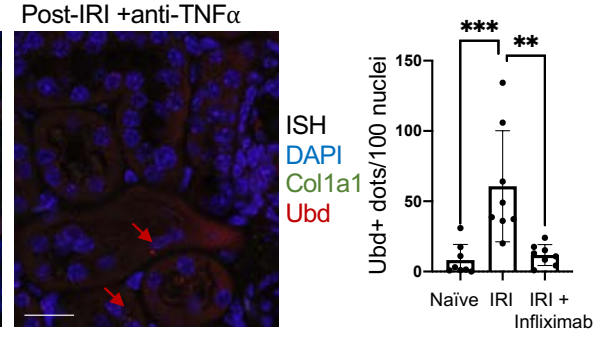

TNF $\alpha$ blockade inhibits Ihh and Col1a1 transcript expression after IRI and UUO injury

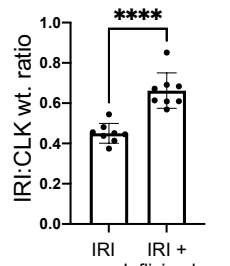

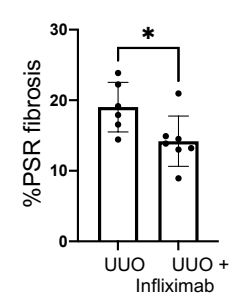

f Schema for study of NFKB inhibition in mice with renal IRI

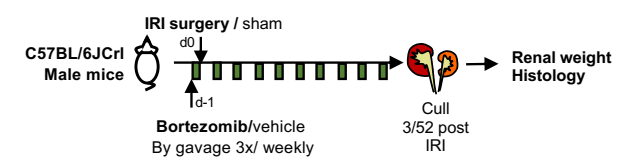

Inhibition of NFKB activation inhibits fibrosis and Ihh transcription after IRI
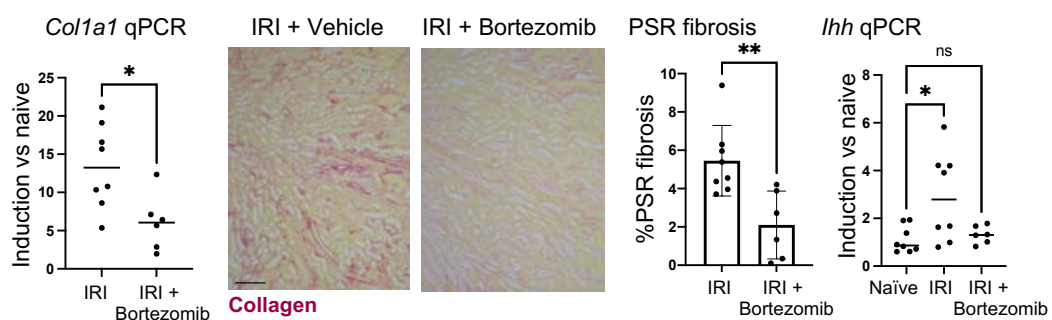
Figure 3. Conditional epithelial deletion of Indian Hedgehog modulates tissue fibrosis in vivo. a. Schema of breeding strategy used to generate Pax8-creERT2; Ihh $\mathrm{fl} / \mathrm{fl}$ mice and confocal images of Colla1, Ubd and Ihh transcripts in healthy and injured kidneys from WT and TG mice b. Schema of experiments testing the impact of Ihh deletion on unilateral ureteric obstruction. c. Ihh $\mathrm{fl} / \mathrm{fl}$ mice treated with tamoxifen show reduced fibrosis compared to WT with tamoxifen, or transgenic without tamoxifen. d. Schema of conditional epithelial restricted Ihh gene deletion before renal IRI. e. Genetic ablation of Ihh increases kidney mass and reduces fibrosis post IRI f. Over-representation analysis illustrates up and downregulated pathways in Ihh deleted kidneys post-IRI. g. UBD and IHH are co-expressed in epithelia from aged and injured human kidneys. h. Serum IHH levels rise in progressive human CKD. Scale bars: 100microns (c), 20 microns (a,g): 5-9/group, h: $\mathrm{n}=12-14$ /group ${ }^{*} \mathrm{p}<0.05, \quad{ }^{*} \mathrm{p}<0.01$, $* * * \mathrm{p}<0.001, * * * * \mathrm{p}<0.0001$ by Student's t-test, one way ANOVA, Kruskal-Wallis test. or Mann-Whitney Test (h). 
a Generation and function of Pax8-creERT2; Ihh h/ffl mice

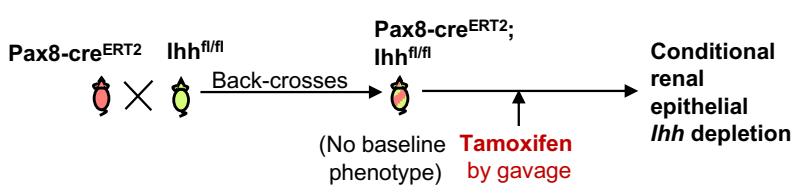

Naïve Kidney

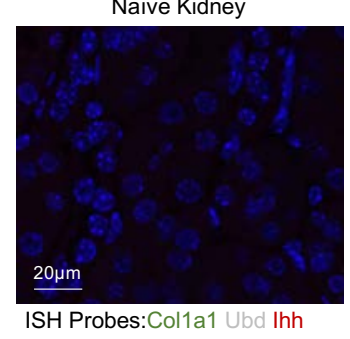

d

Pax8

Pax8-crent2:1nh futtor
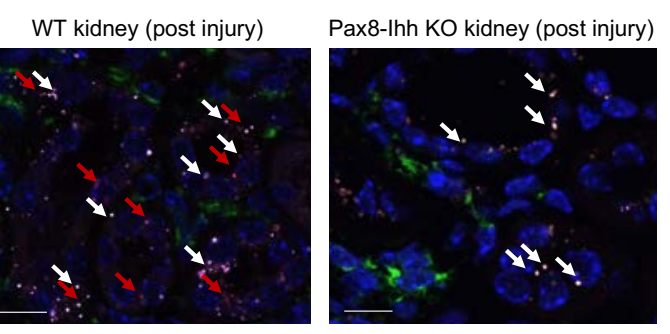

chema for study of conditional renal epithelial Ihh KO pre+post unilateral IRI IRI surgery

$$
\underset{6 / 52 \text { old } \mathrm{d}-21,-19,-17 \mathrm{~d}+7,+9,+11 \text { Cull }}{\longrightarrow} \begin{aligned}
& \text { Renal } \\
& \text { Histology } \\
& \text { RNA sequencing }
\end{aligned}
$$

e Renal size ratio Tamoxifen /vehicle $10 / 52$ post IR

$$
\text { 誌 }
$$

Fibrosis

WT post IRI

Ihh-KO post IRI

$\begin{aligned} \text { IRI } & ++ \\ \text { Ihh KO }-+ & +\end{aligned}$

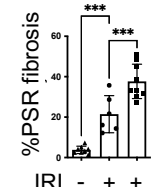

IRI - + +
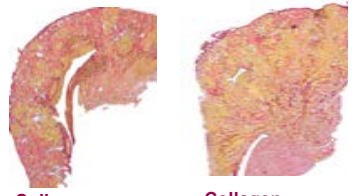

g

$I H H^{\text {ni }} U B D^{\text {hi }}$ cells are present in human aging and CKD

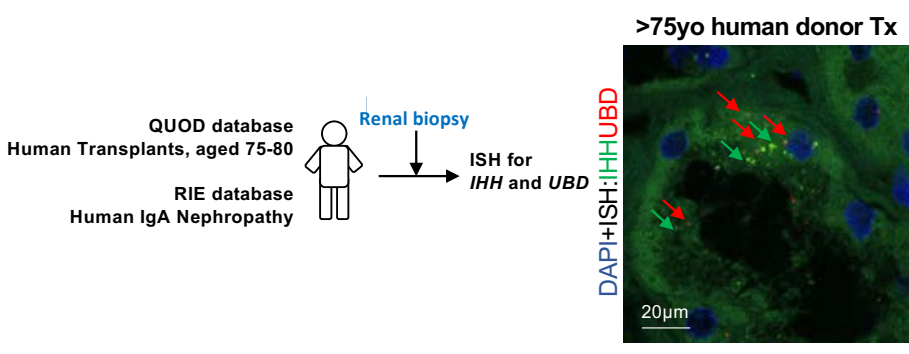

IgA Nephropathy

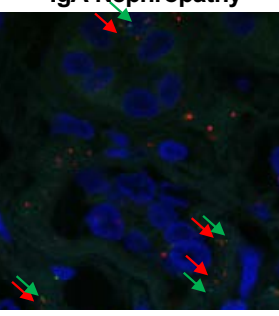

Schema for study of conditional epithelial Ihh depletion in UUO
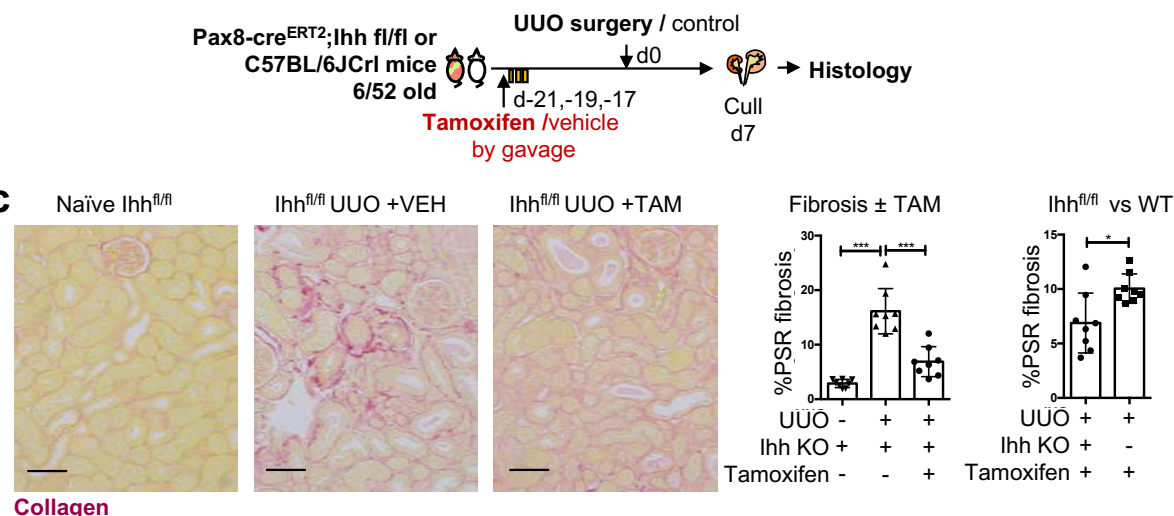

f

ORA plot of Ihh KO vs WT kidneys post-IRI

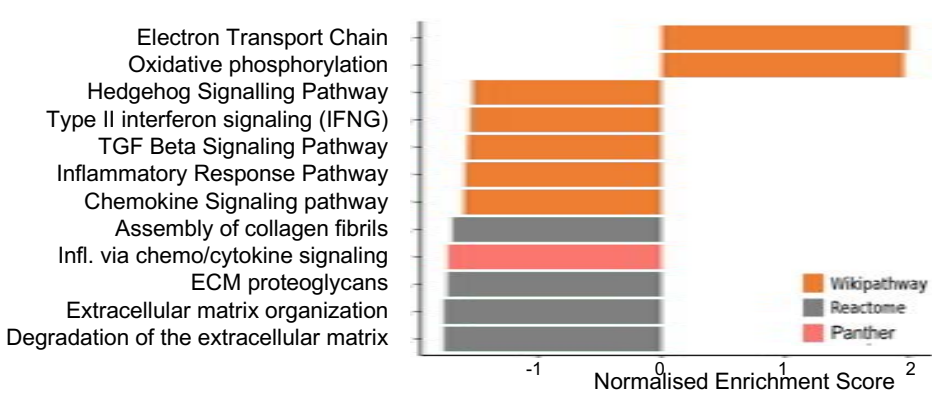

h

Serum IHH levels increase in patients with progressive CKD

seNSOR database Serum sapte Human CKD low risk
or high risk

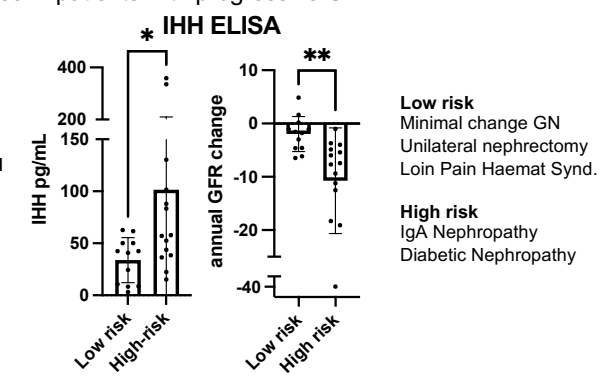




\section{Figure 4. Pharmacological agonism and antagonism of TNF $\alpha / \mathrm{IHH}$ signalling impacts on}

renal fibrosis in vivo. a. Schema of SAG administration to healthy young mice. b. Activation of Hedghog signalling induces fibrosis in the healthy kidney and heart. c. Schema of Hh pathway inhibition studies. d. Smoothened inhibition reduces fibrosis after renal ischaemia. c. Gli inhibition reduces fibrosis after renal ischaemia e. Smoothened inhibition blocks Gli1+ stromal expansion. f. Gli inhibition with GANT61 reduces renal fibrosis on PSR staining g. Gli inhibition with GANT61 reduces collagen transcript expression after injury. h. Schema of postbilateral injury pharmacotherapy studies. i. Picrosirius red staining of collagen in post-IRI kidneys \pm treatment. j. Post-injury TNF $\alpha /$ IHH signalling blockade reduces fibrosis in vivo, but is not additive to Hh deletion. Scale bars: 100 microns. $\mathrm{n}=5-8$ /group. ${ }^{*} \mathrm{p}<0.05,{ }^{*} \mathrm{p}<0.01$, $* * * \mathrm{p}<0.001, * * * * \mathrm{p}<0.0001$ by Student's t-test or ANOVA. 


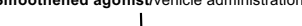

b

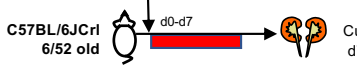
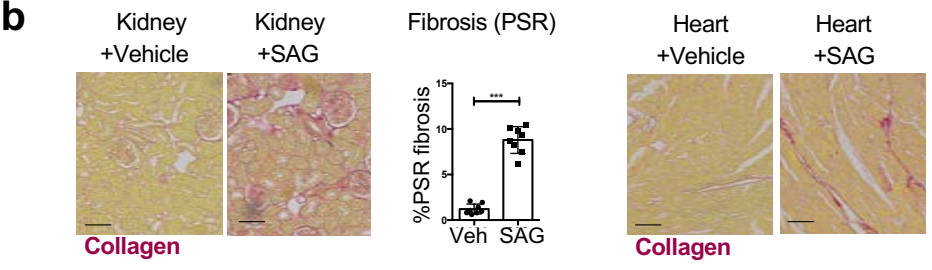

Fibrosis (PSR)

C Hh inhibition reduces post-injury renal fibrosis Gli1 $^{\text {creERT2/TdTom }}{ }^{\text {LSL }}$ or C57BL6/J Unilateral IRI or sham

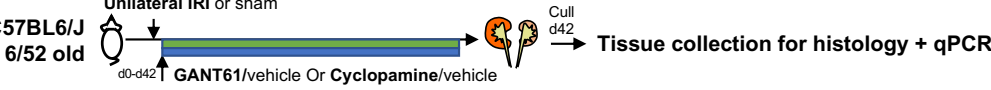

d
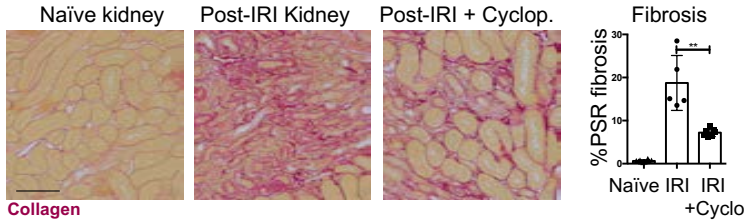

e Naive kidney

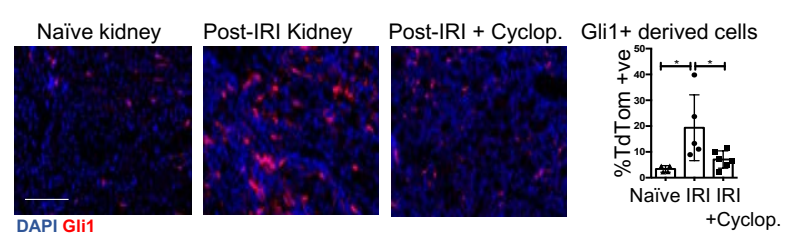

f
Naïve kidney
Post-IRI Kidney Post IRI +GANT61

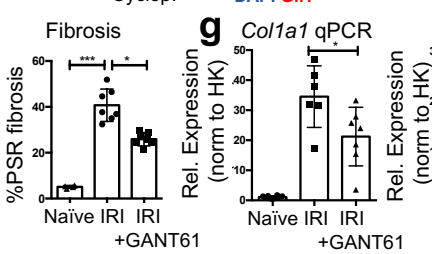

g Col1a1 $\mathrm{qPCR}$

Col3a1 qPCR
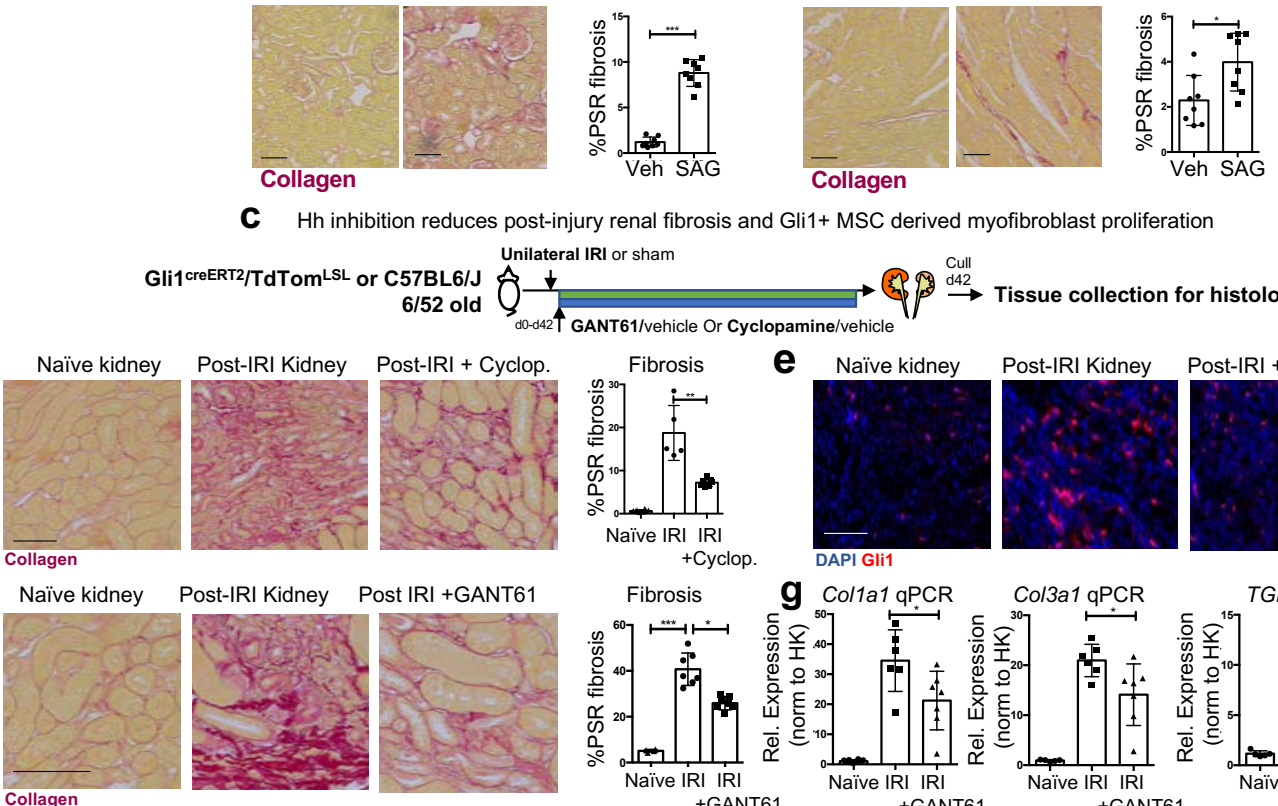

Naive IRI IRI

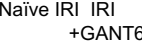

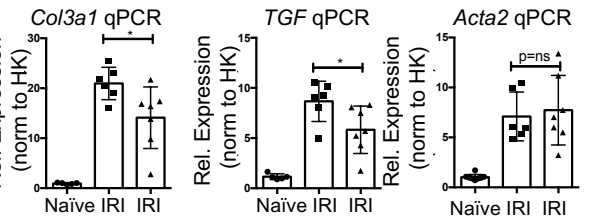

h Inhibition of TNF $\alpha$ or GLI1/2 signalling post injury prevents renal fibrosis and dysfunction, but is not additive to prior epithelial Ihh deletion

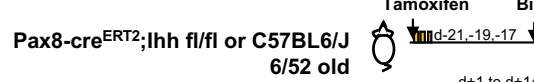
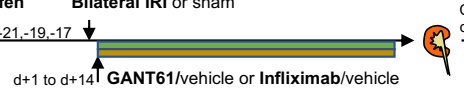
$\stackrel{\substack{\text { Cull } \\ \text { d14 }}}{\longrightarrow}$

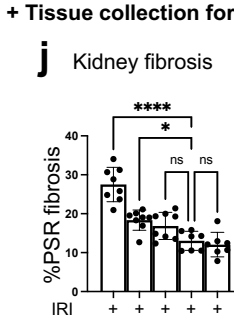
IHH knockdown
Gli1/2 blockade
TNF $\alpha$ blockade $\begin{array}{cc}\text { Post IRI Kidney } & \text { Post IRI Kidney } \\ \text { lhh deletion + Veh } & \text { Inh deletion + Inflix }\end{array}$

Post-IRI Kid
+ Vehicle

Post IRI Kidney
Post IRI Kidney
tpost-op GANT61 $\frac{+ \text { post-op Infliximab }}{20}$

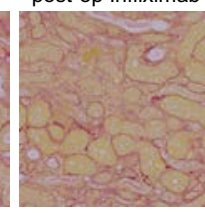

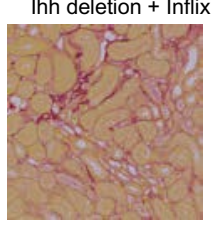

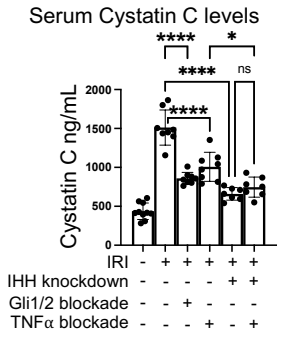


Figure 5. Genetic inhibition or pharmacological antagonism of TNF $\alpha /$ IHH signalling blocks systemic fibrosis after experimental renal injury. a. Schema of conditional epithelial restricted Ihh gene deletion before renal IRI. b. IRI of a single kidney induces cardiac hypertrophy and fibrosis compared to animals lacking renal epithelial Ihh c. Schema of Hh pathway inhibition studies in contralateral kidneys and hearts. d. Hh signalling blockade blocks remote fibrosis in contralateral kidneys after injury. e. Hh signalling blockade blocks remote fibrosis in the heart after kidney injury. Scale bars: 100 microns. $n=4-14 /$ group. ${ }^{*} p<0.05$, ** $<<0.01$, by Kruskal-Wallis test or ANOVA. 
IRI surgery or control

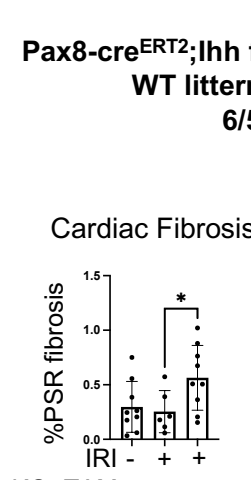

Ihh KO+TAM - + -

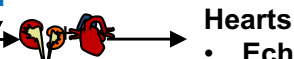

Echocardiography

- Histology

$6 / 52$ old $\mathrm{d}-21,-19,-17 \mathrm{~d}+7,+9,+11$ Cull
Tamoxifen /vehicle 10/52 post IRI

b LV wall thickness

Post IRI Heart (WT)
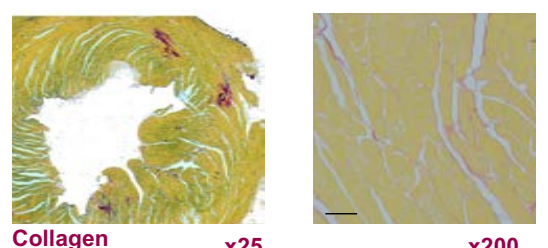

Post-IRI Heart (Ihh KO)
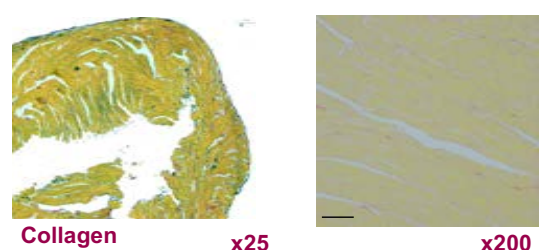

C Hh inhibition reduces contralateral kidney and cardiac fibrosis after unilateral IRI

$$
\text { Gli1 }^{\text {creERT2/TdTom }}{ }^{\text {LSL }} \text { or C57BL6/J }
$$

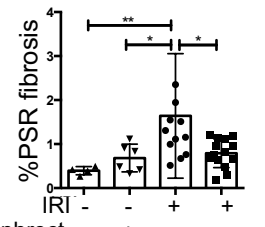

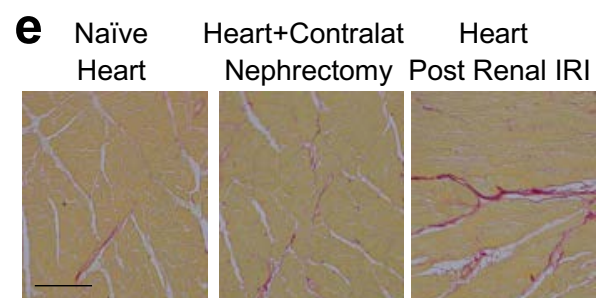

Heart post-IRI

$+\mathrm{Hhi}$

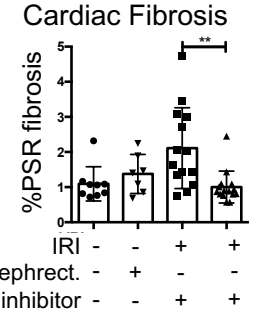


Figure 6. TNF $\alpha$ induces cardiorenal fibrosis via NFKB induced IHH production by iPT cells. Graphical summary of the signalling pathway linking TNF-alpha to organ fibrosis, and points where genetic or pharmacological intervention are antifibrotic in murine models of post-renal injury fibrosis (green).

TNF $\alpha / N F K B$ blockade genetic IHH deletion Smo/Gli inhibitors

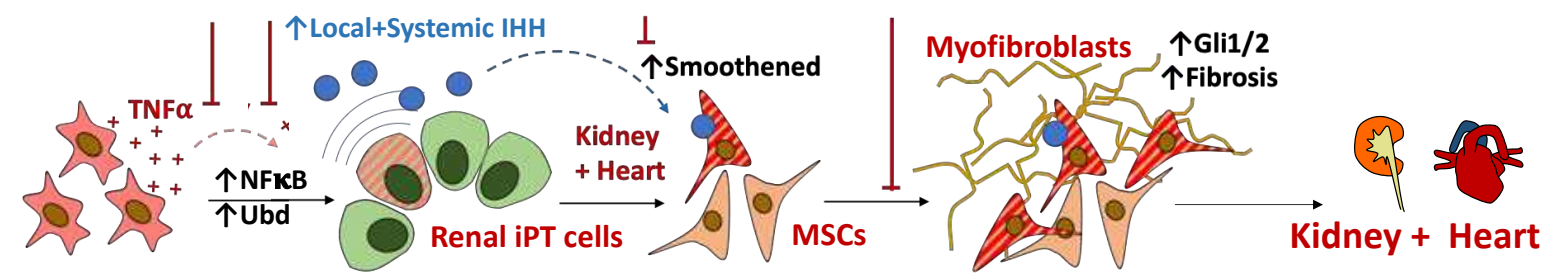

$\begin{array}{lll}\text { Activated } & \text { TNF } \alpha \rightarrow \text { NFKB } & \text { IHH induced myofibroblast } \\ \text { Leukocytes } & \text { induced IHH release } & \text { proliferation and activation }\end{array} \longrightarrow \begin{aligned} & \text { Multiorgan } \\ & \text { Fibrosis }\end{aligned}$




\section{Extended Data Figures}

Extended Data Figure 1. UMAP plots of young uninjured and fibrotic kidneys 6/52 post

ischaemic injury. a. Uninjured young kidney b. fibrotic kidney 6/52 post injury, both showing clusters and differentially expressed genes on single cell RNA sequencing. ( $n=3$ /group). 
a UMAP plots from young $\mathbf{C}$ healthy kidneys

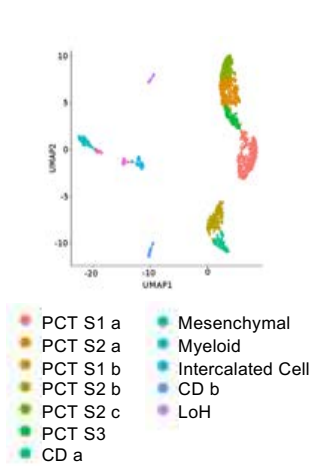

b UMAP plots from fibrotic kidneys $6 / 52$ post injury io is a c is

ก.

$\because \& \&$

is

$\because$ PCTall : Macb

Endothelial : Mesenchyma

Mac a $\mathrm{LOH}$

$\because \begin{array}{ll}\text { CDa } \\ \text { DCT a }\end{array}$
Cluster heatmaps from young healthy kidneys

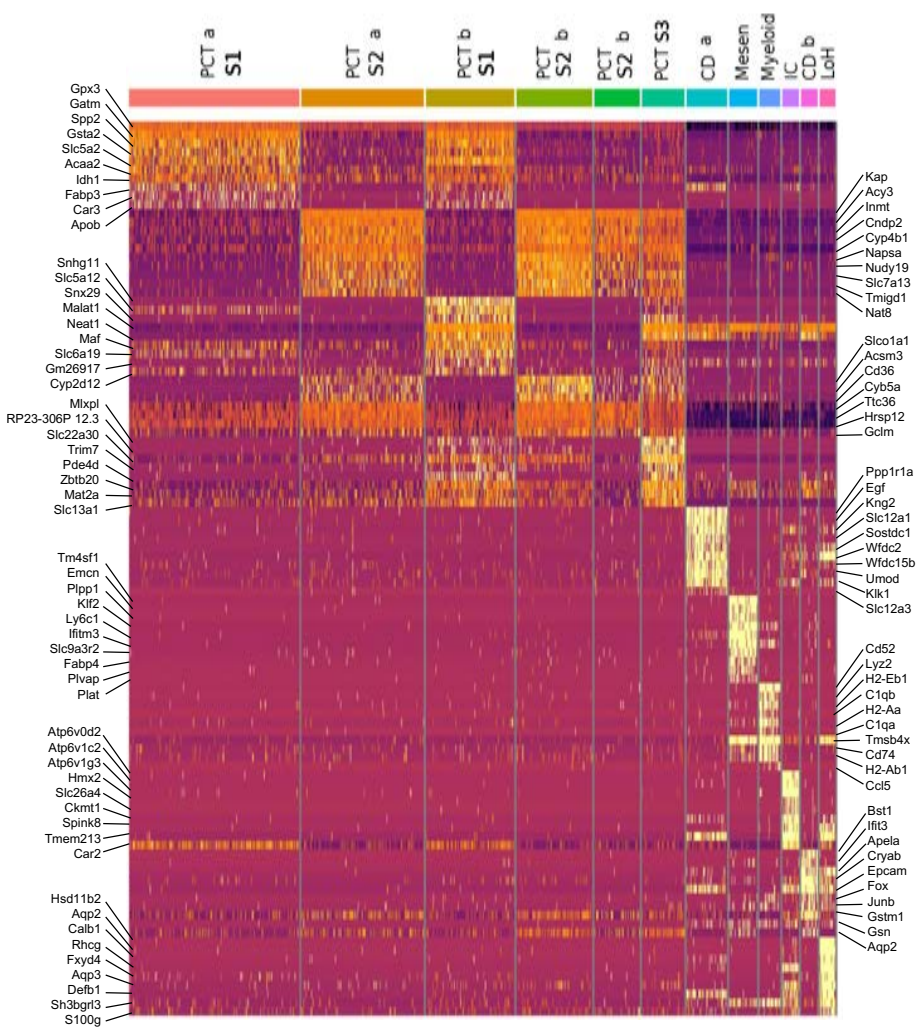

d Cluster heatmaps from young fibrotic kidneys 6/52 post injury

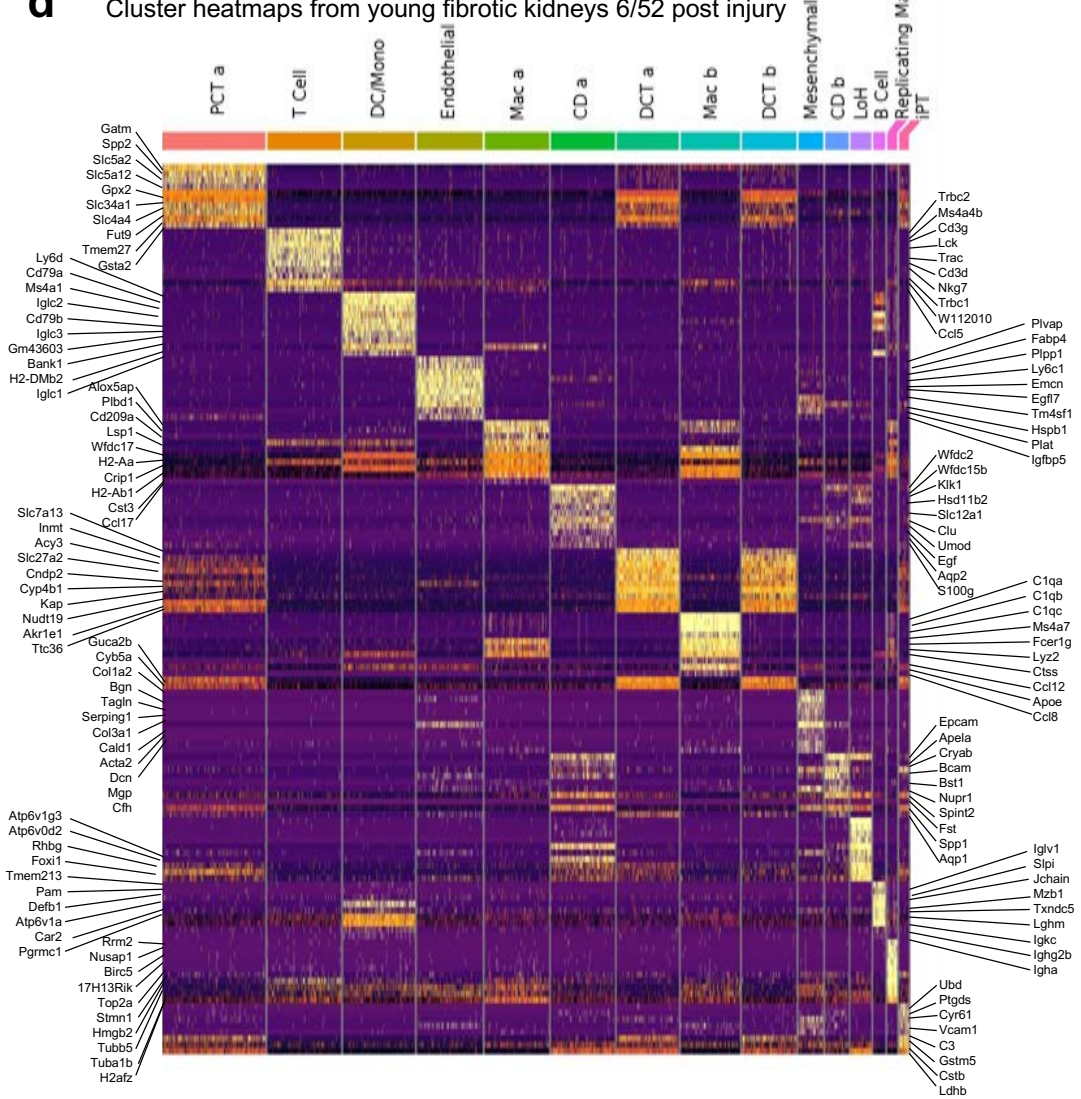


Extended Data Figure 2. Tubular epithelial marker genes and epithelial cell heatmaps of uninjured young, fibrotic young and aged kidneys. a. Uninjured young kidney b. fibrotic kidney 6/52 post injury, c. TMS old and young kidneys, all showing tubular marker genes and differentially expressed genes for epithelial clustering on single cell RNA sequencing. ( $n=3 /$ group $)$. 
a Dotplot of tubular marker genes in the young healthy kidney

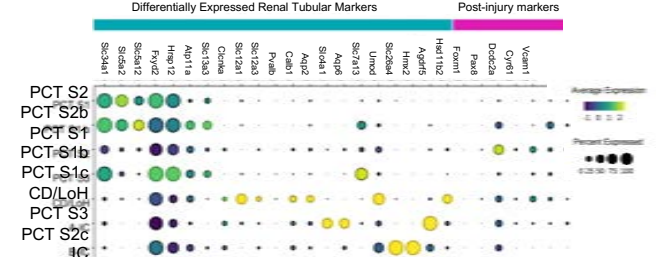

Heatmap of epithelial clusters in the young healthy kidney

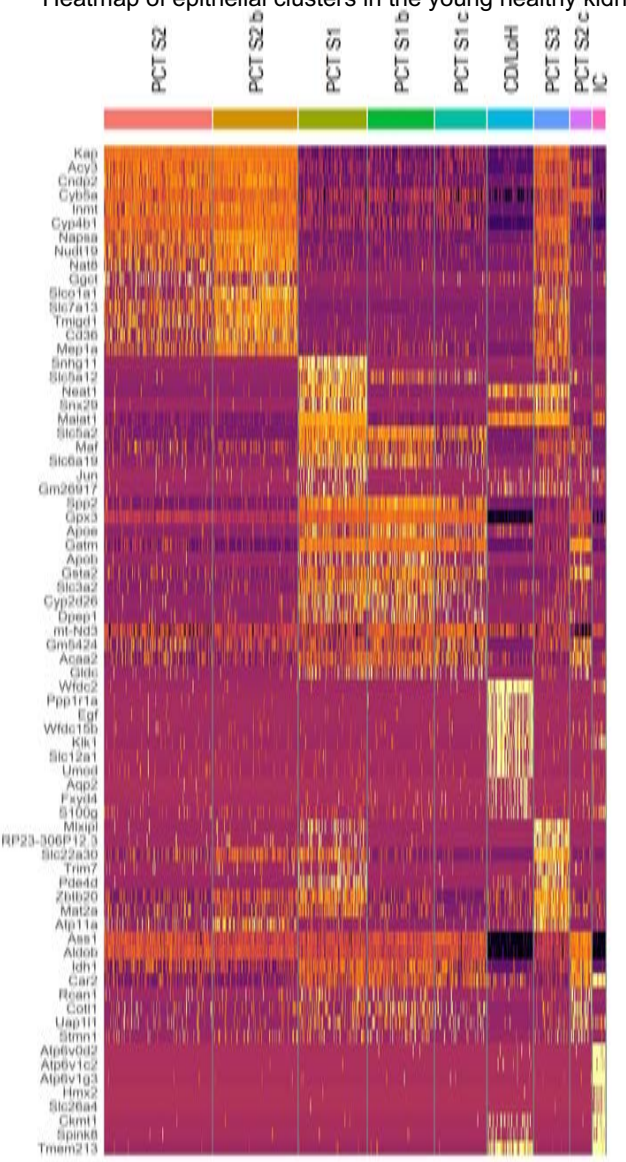

D Dotplot of tubular marker genes in the young fibrotic kidney

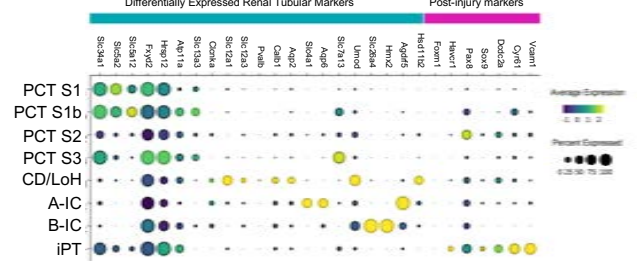

Heatmap of epithelial clusters in the young fibrotic kidney

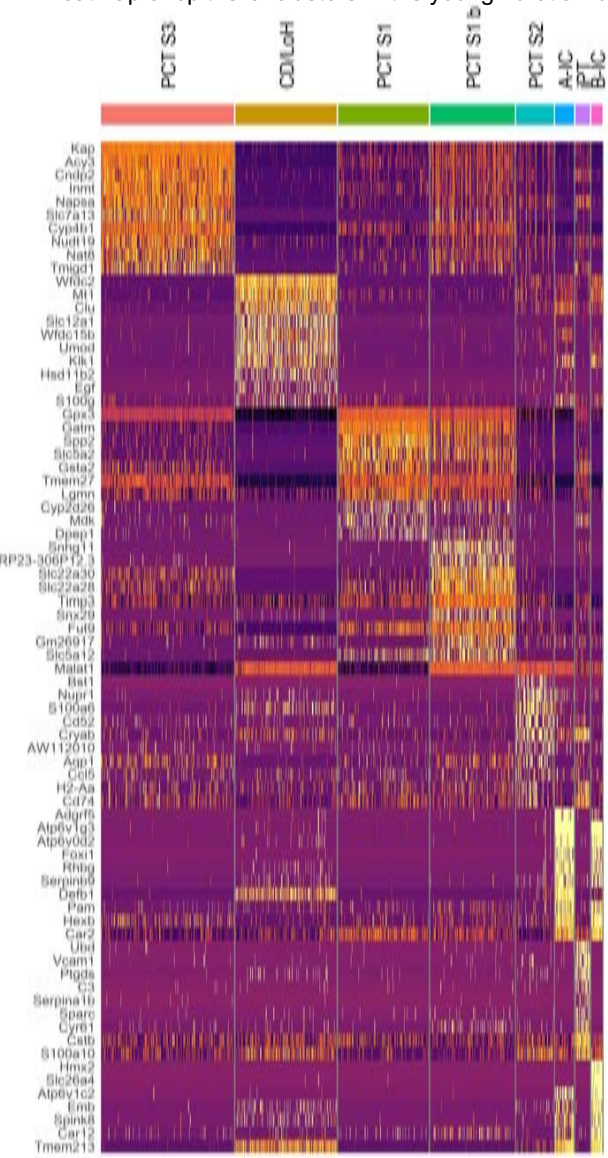

c Dotplot of tubular marker genes in the TMS kidney dataset

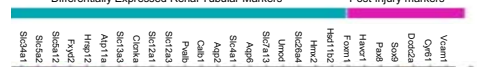
PCT S1
PCT S2
PCo

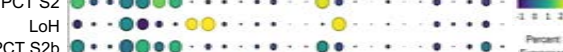
DCT CD
PCT S3
P

Heatmap of epithelial clusters in the young fibrotic kidney

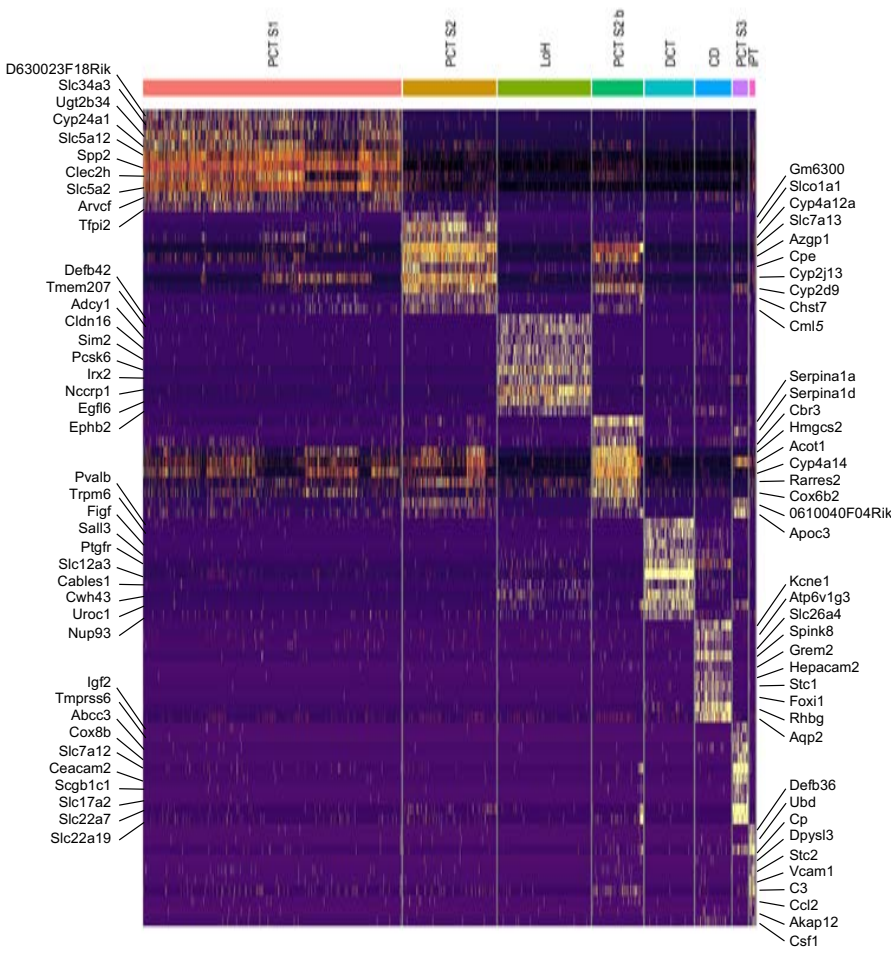


Extended Data Figure 3. Expression of UBD in human ageing and kidney fibrosis. Work by Rodwell at al shows a significant association between Ubd expression and age and fibrosis/atrophy in human kidney biopsies. $(n=74)$

Renal UBD, a marker of iPT cells rises with age and with markers of fibrosis/atrophy

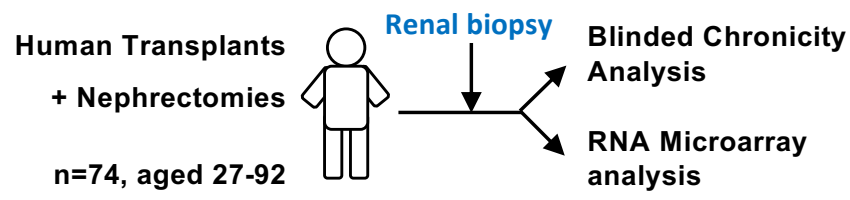

Renal Ubd vs age

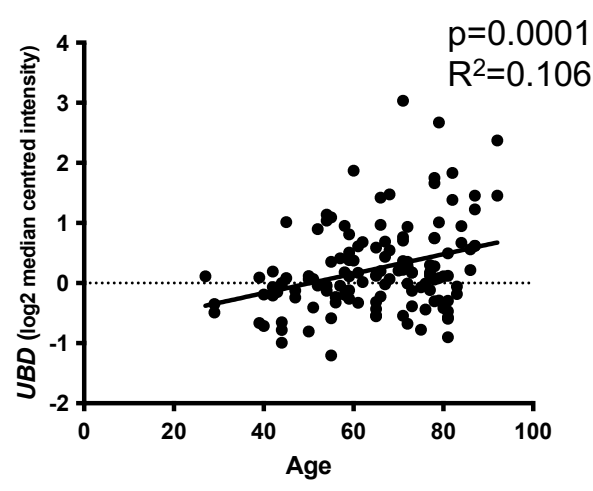

Renal Ubd vs fibrosis quartile

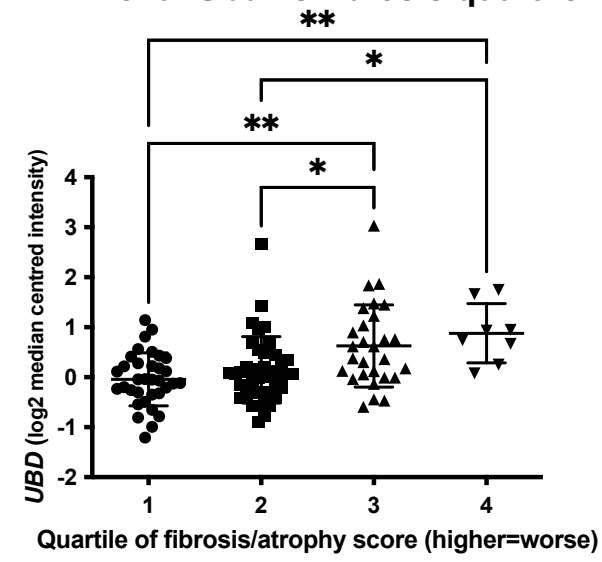

Rodwell et al. PLoS Biol 2004 
Extended Data Figure 4. Identification of Tnf transcripts within and control/fibrotic kidney UMAP plots. Identification of $\operatorname{Tn} f$ transcripts in uninjured and post-injured fibrotic kidneys.

UMAP plots of fibrotic kidneys in uninjured and post-IRI kidneys show renal Tnf expression restricted to Myeloid and Lymphoid leukocytes
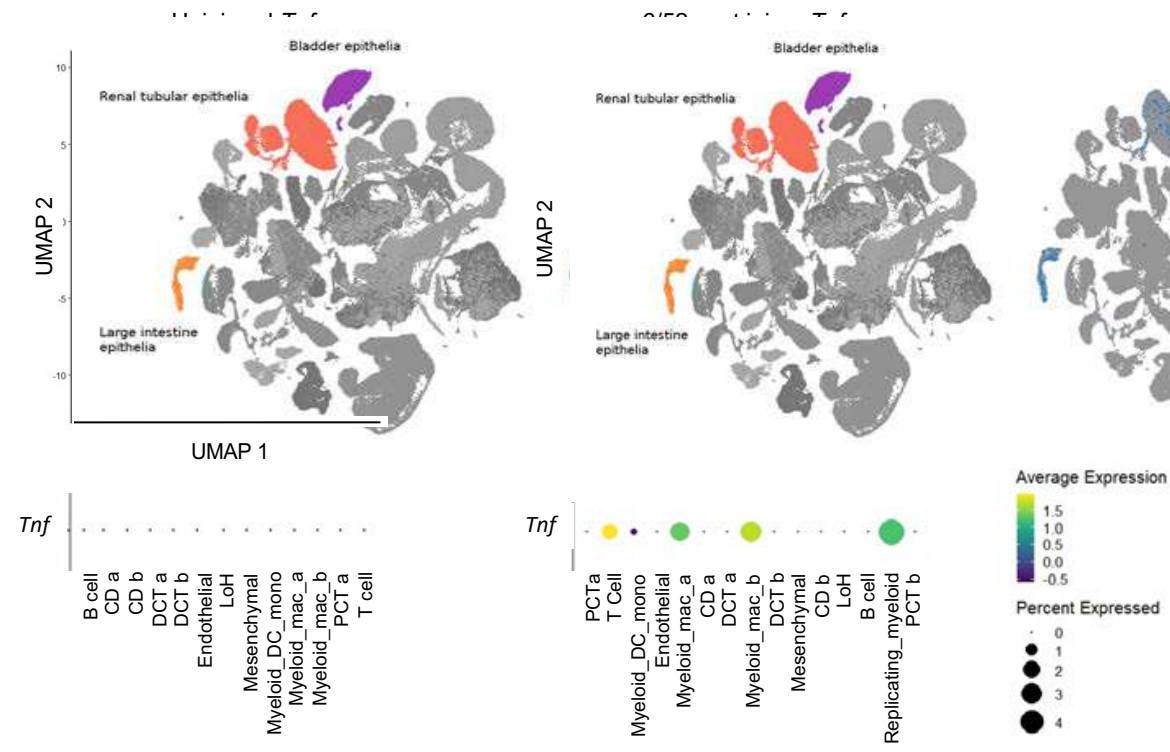
Extended Data Figure 5. TNF $\alpha$ induces $U b d$ in human RPTECs in vitro. a. Schema of studies of TNF $\alpha$ administration to human proximal tubular cells in vitro b. TNF $\alpha$ induces $U b d$ in the presence of a functional NFאB pathway. ${ }^{* * *} \mathrm{p}<0.001 . \mathrm{n}=3$ /group. Kruskal-Wallis Test.

a Schema for TNF $\alpha$ blockade in vitro

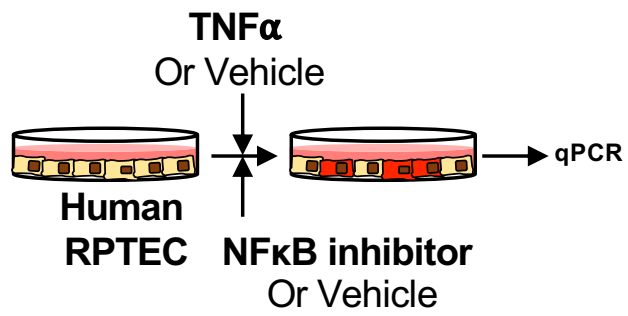

b TNF $\alpha$ induces RPTEC Ubd in vitro

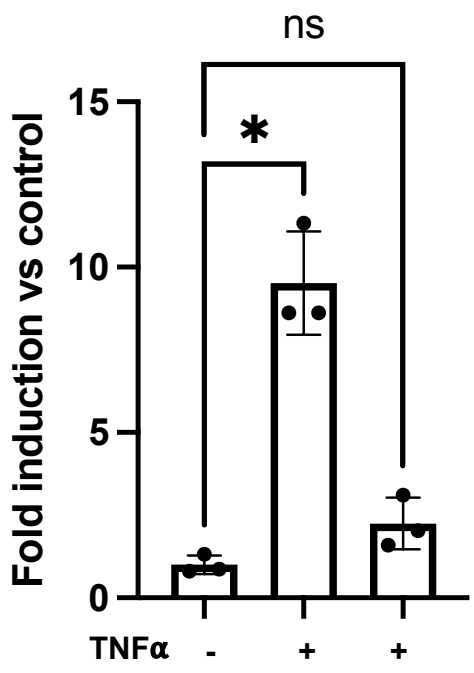

NFKB inhibitor 
Extended Data Figure 6. Identification of $S h h$ and $I h h$ transcripts in multi-organ TMS UMAP plot

TMS droplet based sequencing shows bladder vs kidney restriction of Shh and Ihh transcripts

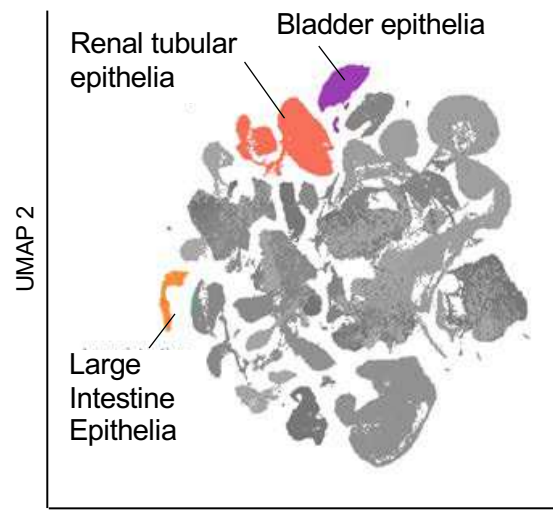

UMAP 1

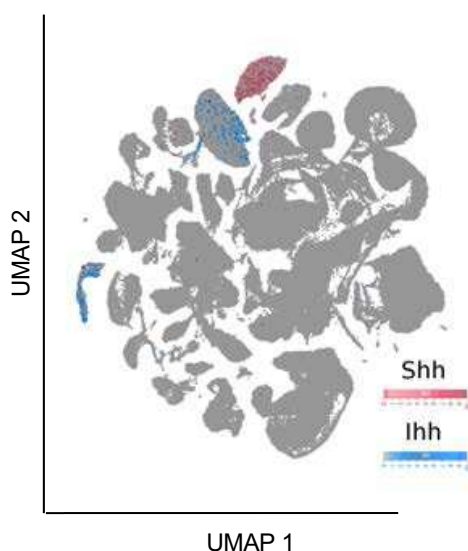




\section{Extended Data Figure 7. iPT markers correlate with fibrosis induction in reversed UUO}

and are present with ageing. Induction of Hedgehog signalling is sufficient to induce cardiac and renal fibrosis in vitro and in vivo. a. Schema of studies using murine reversed UUO b. RNAseq shows early induction of Ubd and Ihh. n=4/group. c. Ubd and Ihh co-localize in tubular epithelial cells from 18/12 old murine kidneys. d. Schema of studies testing recombinant IHH and Smoothened Agonist (SAG) administration to human renal fibroblasts. e. IHH and SAG both induce fibrotic activation of fibroblasts in vitro.

a Study of transcriptomic changes in UUO and reversed UUO (Conway et al 2020)

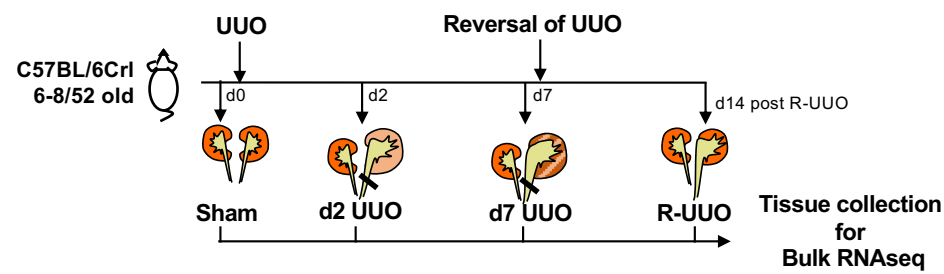

b Results of differentially expressed genes in Bulk RNAseq analysis of naïve vs UUO vs R-UUO kidneys

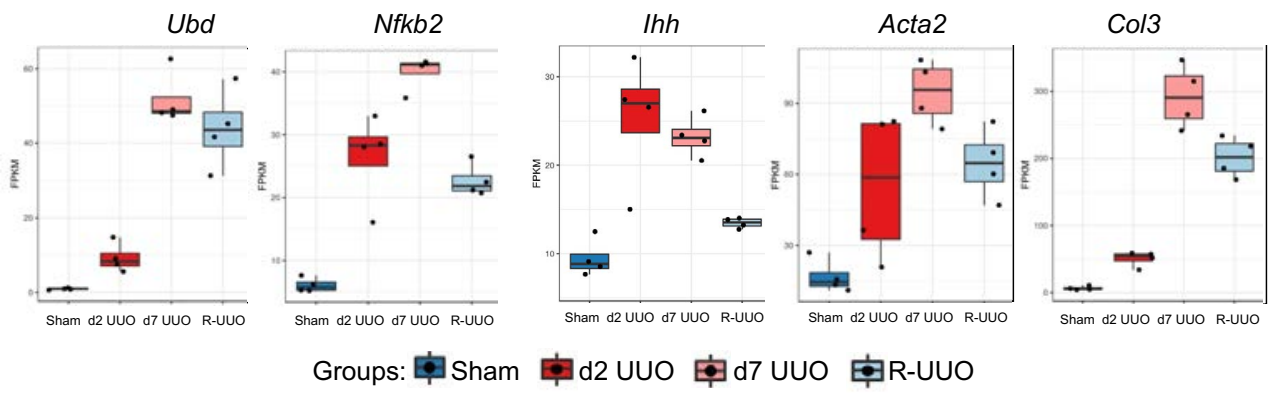

C $U b d+I h h$ colocalize in IPT cells with aging

d Schema for Hh pathway activation in vitro

e Ihh activates human fibroblasts in vitro

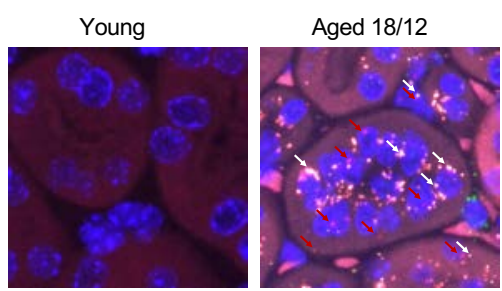

ISH Stains: DAPI Col1a1 Ubd Ihh

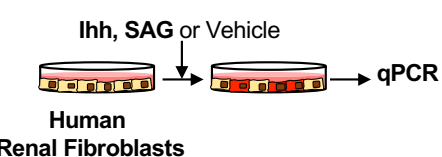

Renal Fibroblasts

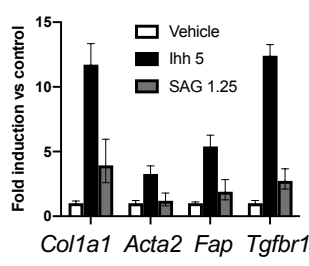


Extended Data Figure 8. Healthy young human kidney expresses low levels of epithelial UBD and IHH a. Human kidney tissue from young donors (aged $<20$ ) shows minimal staining for IHH or UBD transcripts. Old kidney tissue staining with negative control probes shows no positive staining (Scale bar: 20 micron, both images shown at +10 Contrast and +60 Brightness on Microsoft Powerpoint).

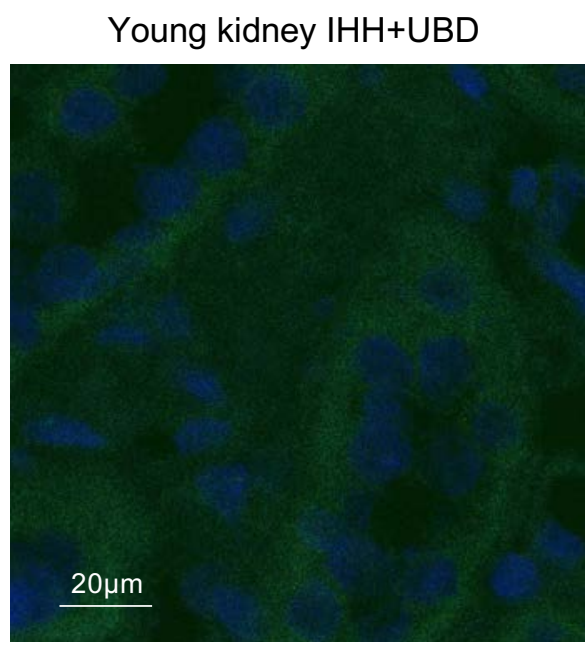

Old Kidney negative controls

DAPI + ISH Probes: IHH UBD 
Extended Data Figure 9. The presence of a fibrotic kidney leads to upregulated inflammatory and fibrotic signalling in the uninjured contralateral kidney. Heatmap of key DEG between kidneys contralateral to a nephrectomy compared to kidneys contralateral to IRI ( $\mathrm{n}=3-4 /$ group). calculated using Wilcoxon signed-rank test. The colour scheme is based on z-score distribution, all comparisons are significant padj $<0.1$

$\begin{array}{cc}\text { Uninjured kidney } & \text { Uninjured kidney } \\ \text { (contralateral nephrectomy) } & \text { (IRI to Contralateral 10/52 previous) }\end{array}$

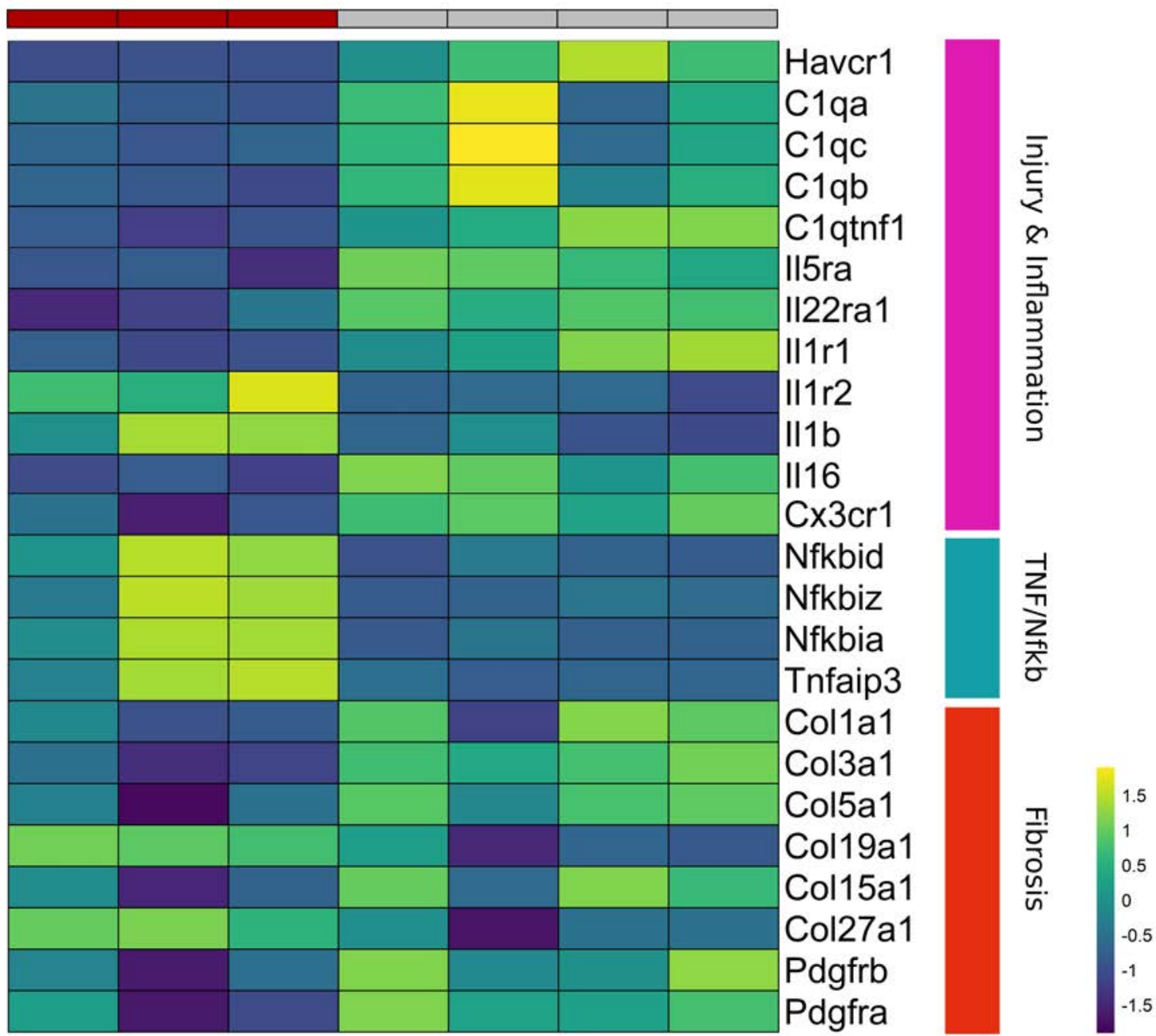


Extended Data Figure 10. a. Despite a lack of endogenous $I h h$ or $S h h$ expression, multiple markers of Hedgehog activation are elevated in the TMS murine heart dataset, with no expression of Pax 8 detected (purple dots represent no detectable transcript across entire dataset). b. Murine blood pressures are not significantly elevated in any intervention group after unilateral IRI surgery. 
a Expression of $\mathrm{Hh}$ ligands and signaling in murine hearts demonstrates multiple markers of Hedgehog signalling with no native Pax8 or Shh, and minimal Ihh expression

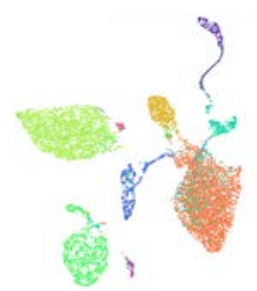

$\begin{array}{llll}\text { fibroblast } & =2372 & \text { atrial cardiomyocyte } & \square 294 \\ \text { leukocyte } & =908 & \text { cardiac mast cell } & =57 \\ \text { nan } & =964 & \text { cardiac neuron } & \square 44 \\ \text { red blood cell } & =121 & \text { coronary vascular endothelial cell } & =2946 \\ \text { smooth muscle cell } & =373 & \text { endocardial endothelial cells } & =534\end{array}$
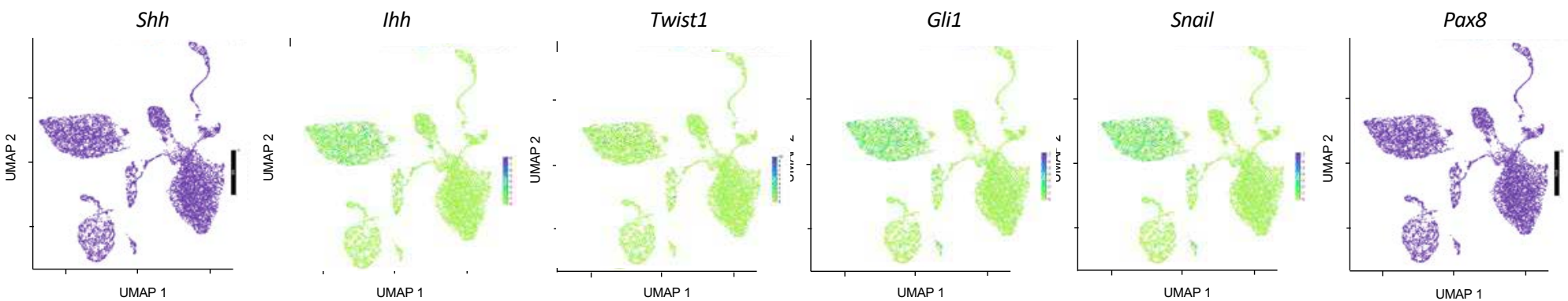

b

Pre and 10 weeks post IRI BP

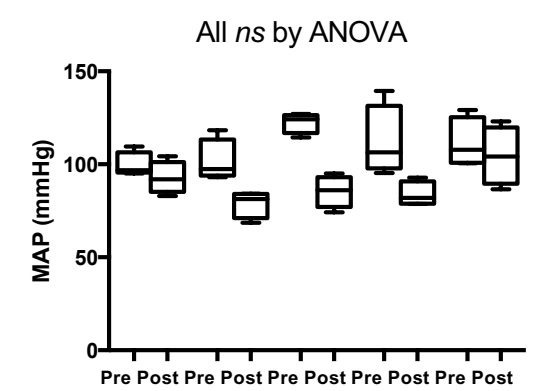

$\begin{array}{rccccc}\mathrm{IRI} & + & + & + & - & - \\ \mathrm{Ihh} \mathrm{KO} & + & - & + & - & - \\ \text { Tamoxifen } & + & + & - & - & - \\ \text { Nephrectomy } & - & - & - & + & -\end{array}$





\section{References}

1 Goronzy, J. J. \& Weyand, C. M. Successful and Maladaptive T Cell Aging. Immunity 46, 364-378, doi:10.1016/j.immuni.2017.03.010 (2017).

2 Nikolich-Zugich, J. The twilight of immunity: emerging concepts in aging of the immune system. Nat Immunol 19, 10-19, doi:10.1038/s41590-017-0006-x (2018).

3 Hearps, A. C. et al. Aging is associated with chronic innate immune activation and dysregulation of monocyte phenotype and function. Aging cell 11, 867-875, doi:10.1111/j.1474-9726.2012.00851.x (2012).

4 Santos Morais Junior, G. et al. Circulating Interleukin-6 (but Not Other Immune Mediators) Associates with Criteria for Fried's Frailty among Very Old Adults. J Aging Res 2020, 6831791, doi:10.1155/2020/6831791 (2020).

5 Gencer, S., Evans, B. R., van der Vorst, E. P. C., Doring, Y. \& Weber, C. Inflammatory Chemokines in Atherosclerosis. Cells 10, doi:10.3390/cells10020226 (2021).

6 Kurts, C., Panzer, U., Anders, H. J. \& Rees, A. J. The immune system and kidney disease: basic concepts and clinical implications. Nat Rev Immunol 13, 738-753, doi:10.1038/nri3523 (2013).

7 Sato, Y. \& Yanagita, M. Immunology of the ageing kidney. Nat Rev Nephrol 15, 625640, doi:10.1038/s41581-019-0185-9 (2019).

8 Jankowski, J., Floege, J., Fliser, D., Bohm, M. \& Marx, N. Cardiovascular Disease in Chronic Kidney Disease: Pathophysiological Insights and Therapeutic Options. Circulation 143, 1157-1172, doi:10.1161/CIRCULATIONAHA.120.050686 (2021).

9 Nikolic-Paterson, D. J., Wang, S. \& Lan, H. Y. Macrophages promote renal fibrosis through direct and indirect mechanisms. Kidney international supplements 4, 34-38, doi:10.1038/kisup.2014.7 (2014).

10 Eardley, K. S. et al. The role of capillary density, macrophage infiltration and interstitial scarring in the pathogenesis of human chronic kidney disease. Kidney Int 74, 495-504, doi:10.1038/ki.2008.183 (2008).

11 Nelson, P. J. et al. The renal mononuclear phagocytic system. J Am Soc Nephrol 23, 194-203, doi:10.1681/ASN.2011070680 (2012).

12 Seo, E. et al. The Hippo-Salvador signaling pathway regulates renal tubulointerstitial fibrosis. Sci Rep 6, 31931, doi:10.1038/srep31931 (2016).

13 Bera, A. et al. Reciprocal regulation of miR-214 and PTEN by high glucose regulates renal glomerular mesangial and proximal tubular epithelial cell hypertrophy and matrix expansion. American journal of physiology. Cell physiology 313, C430-C447, doi:10.1152/ajpcell.00081.2017 (2017).

14 Du, R. et al. Hypoxia-induced down-regulation of microRNA-34a promotes EMT by targeting the Notch signaling pathway in tubular epithelial cells. PLoS One 7, e30771, doi:10.1371/journal.pone.0030771 (2012).

15 Tabula Muris, C. A single-cell transcriptomic atlas characterizes ageing tissues in the mouse. Nature 583, 590-595, doi:10.1038/s41586-020-2496-1 (2020).

16 Gong, P. et al. The ubiquitin-like protein FAT10 mediates NF-kappaB activation. J Am Soc Nephrol 21, 316-326, doi:10.1681/ASN.2009050479 (2010).

17 Ross, M. J. et al. Role of ubiquitin-like protein FAT10 in epithelial apoptosis in renal disease. J Am Soc Nephrol 17, 996-1004, doi:10.1681/ASN.2005070692 (2006).

18 Jun, J. I. \& Lau, L. F. Taking aim at the extracellular matrix: CCN proteins as emerging therapeutic targets. Nat Rev Drug Discov 10, 945-963, doi:10.1038/nrd3599 (2011). 

redefine cellular heterogeneity in the adult human kidney. Nature communications 12, 2190, doi:10.1038/s41467-021-22368-w (2021).

20 Rodwell, G. E. et al. A transcriptional profile of aging in the human kidney. PLoS biology 2, e427, doi:10.1371/journal.pbio.0020427 (2004).

21 Abel, S. et al. The transmembrane CXC-chemokine ligand 16 is induced by IFNgamma and TNF-alpha and shed by the activity of the disintegrin-like metalloproteinase ADAM10. J Immunol 172, 6362-6372, doi:10.4049/jimmunol.172.10.6362 (2004).

22 Perrot-Applanat, M. et al. Similar NF-kappaB gene signatures in TNF-alpha treated human endothelial cells and breast tumor biopsies. PLoS One 6, e21589, doi:10.1371/journal.pone.0021589 (2011).

23 Mehaffey, E. \& Majid, D. S. A. Tumor necrosis factor-alpha, kidney function, and hypertension. Am J Physiol Renal Physiol 313, F1005-F1008, doi:10.1152/ajprenal.00535.2016 (2017).

24 Edeling, M., Ragi, G., Huang, S., Pavenstadt, H. \& Susztak, K. Developmental signalling pathways in renal fibrosis: the roles of Notch, Wnt and Hedgehog. Nat Rev Nephrol 12, 426-439, doi:10.1038/nrneph.2016.54 (2016).

25 Zhou, D. et al. Sonic hedgehog is a novel tubule-derived growth factor for interstitial fibroblasts after kidney injury. J Am Soc Nephrol 25, 2187-2200, doi:10.1681/ASN.2013080893 (2014).

26 Conway, B. R. et al. Kidney Single-Cell Atlas Reveals Myeloid Heterogeneity in Progression and Regression of Kidney Disease. J Am Soc Nephrol 31, 2833-2854, doi:10.1681/ASN.2020060806 (2020).

27 Daoussis, D. et al. Anti-TNFalpha treatment decreases the previously increased serum Indian Hedgehog levels in patients with ankylosing spondylitis and affects the expression of functional Hedgehog pathway target genes. Semin Arthritis Rheum 44, 646-651, doi:10.1016/j.semarthrit.2015.01.004 (2015).

28 Kramann, R. et al. Pharmacological GLI2 inhibition prevents myofibroblast cell-cycle progression and reduces kidney fibrosis. J Clin Invest 125, 2935-2951, doi:10.1172/JCI74929 (2015).

29 Kramann, R. et al. Perivascular Gli1+ progenitors are key contributors to injuryinduced organ fibrosis. Cell stem cell 16, 51-66, doi:10.1016/j.stem.2014.11.004 (2015).

30 Coca, S. G., Singanamala, S. \& Parikh, C. R. Chronic kidney disease after acute kidney injury: a systematic review and meta-analysis. Kidney Int 81, 442-448, doi:10.1038/ki.2011.379 (2012).

31 Arcari, L. et al. Cardiac biomarkers in chronic kidney disease are independently associated with myocardial edema and diffuse fibrosis by cardiovascular magnetic resonance. J Cardiovasc Magn Reson 23, 71, doi:10.1186/s12968-021-00762-z (2021).

32 Yoshida, Y. et al. Alteration of circadian machinery in monocytes underlies chronic kidney disease-associated cardiac inflammation and fibrosis. Nature communications 12, 2783, doi:10.1038/s41467-021-23050-x (2021).

33 Charytan, D. M. et al. Increased concentration of circulating angiogenesis and nitric oxide inhibitors induces endothelial to mesenchymal transition and myocardial 
fibrosis in patients with chronic kidney disease. International journal of cardiology 176, 99-109, doi:10.1016/j.ijcard.2014.06.062 (2014).

34 Kawagishi, H. et al. Sonic hedgehog signaling regulates the mammalian cardiac regenerative response. Journal of molecular and cellular cardiology 123, 180-184, doi:10.1016/j.yjmcc.2018.09.005 (2018).

35 Kusano, K. F. et al. Sonic hedgehog myocardial gene therapy: tissue repair through transient reconstitution of embryonic signaling. Nature medicine 11, 1197-1204, doi:10.1038/nm1313 (2005).

36 Song, K. et al. PDGFRA in vascular adventitial MSCs promotes neointima formation in arteriovenous fistula in chronic kidney disease. JCl Insight 5, doi:10.1172/jci.insight.137298 (2020).

37 Collaboration, G. B. D. C. K. D. Global, regional, and national burden of chronic kidney disease, 1990-2017: a systematic analysis for the Global Burden of Disease Study 2017. Lancet 395, 709-733, doi:10.1016/S0140-6736(20)30045-3 (2020).

38 Coresh, J. et al. Prevalence of chronic kidney disease in the United States. JAMA 298, 2038-2047, doi:10.1001/jama.298.17.2038 (2007).

39 Zhang, J. et al. Tumor necrosis factor-alpha produced in the kidney contributes to angiotensin II-dependent hypertension. Hypertension 64, 1275-1281, doi:10.1161/HYPERTENSIONAHA.114.03863 (2014).

40 Dunaeva, M. \& Waltenberger, J. Hh signaling in regeneration of the ischemic heart. Cell Mol Life Sci 74, 3481-3490, doi:10.1007/s00018-017-2534-9 (2017).

41 Ding, H. et al. Sonic hedgehog signaling mediates epithelial-mesenchymal communication and promotes renal fibrosis. J Am Soc Nephrol 23, 801-813, doi:10.1681/ASN.2011060614 (2012).

42 Kumar, S. et al. Sox9 Activation Highlights a Cellular Pathway of Renal Repair in the Acutely Injured Mammalian Kidney. Cell Rep 12, 1325-1338, doi:10.1016/j.celrep.2015.07.034 (2015).

43 Oh, J. H. et al. A novel cyclin-dependent kinase inhibitor down-regulates tumor necrosis factor-alpha (TNF-alpha)-induced expression of cell adhesion molecules by inhibition of NF-kappaB activation in human pulmonary epithelial cells. International immunopharmacology 10, 572-579, doi:10.1016/j.intimp.2010.02.004 (2010).

44 Sumida, K. et al. Treatment of rheumatoid arthritis with biologic agents lowers the risk of incident chronic kidney disease. Kidney Int 93, 1207-1216, doi:10.1016/j.kint.2017.11.025 (2018).

$45 \mathrm{Kim}, \mathrm{H}$. W. et al. Effect of anti-tumor necrosis factor alpha treatment of rheumatoid arthritis and chronic kidney disease. Rheumatol Int 35, 727-734, doi:10.1007/s00296014-3146-4 (2015).

46 Nurmohamed, M. et al. Longer durations of antitumour necrosis factor treatment are associated with reduced risk of cardiovascular events in patients with rheumatoid arthritis. RMD Open 1, e000080, doi:10.1136/rmdopen-2015-000080 (2015).

47 Kilkenny, C., Browne, W. J., Cuthill, I. C., Emerson, M. \& Altman, D. G. Improving bioscience research reporting: the ARRIVE guidelines for reporting animal research. PLoS biology 8, e1000412, doi:10.1371/journal.pbio.1000412 (2010).

48 Ferenbach, D. A. et al. The induction of macrophage hemeoxygenase- 1 is protective during acute kidney injury in aging mice. Kidney Int 79, 966-976, doi:ki2010535 [pii] 10.1038/ki.2010.535 (2011). 
49 Kipari, T. et al. Nitric oxide is an important mediator of renal tubular epithelial cell death in vitro and in murine experimental hydronephrosis. Am J Pathol 169, 388-399, doi:169/2/388 [pii] (2006).

50 Wang, F. et al. RNAscope: a novel in situ RNA analysis platform for formalin-fixed, paraffin-embedded tissues. J Mol Diagn 14, 22-29, doi:10.1016/j.jmoldx.2011.08.002 (2012).

51 Bankhead, P. et al. QuPath: Open source software for digital pathology image analysis. Sci Rep 7, 16878, doi:10.1038/s41598-017-17204-5 (2017).

52 Dobin, A. et al. STAR: ultrafast universal RNA-seq aligner. Bioinformatics 29, 15-21, doi:10.1093/bioinformatics/bts635 (2013).

53 Butler, A., Hoffman, P., Smibert, P., Papalexi, E. \& Satija, R. Integrating single-cell transcriptomic data across different conditions, technologies, and species. Nature biotechnology 36, 411-420, doi:10.1038/nbt.4096 (2018).

54 Ilicic, T. et al. Classification of low quality cells from single-cell RNA-seq data. Genome biology 17, 29, doi:10.1186/s13059-016-0888-1 (2016). 